\title{
Ecology and distribution of soft-sediment benthic communities off Viti Levu (Fiji)
}

\author{
Shirley W. C. Mohammed ${ }^{1, *}$, Simon E. Coppard ${ }^{2,3}$ \\ ${ }^{1}$ Marine Studies Program, University of the South Pacific, Laucala Campus, Suva, Fiji \\ ${ }^{2}$ Department of Zoology, Natural History Museum, Cromwell Road, London SW7 5BD, UK \\ ${ }^{3}$ Smithsonian Tropical Research Institute, Balboa, Panama
}

\begin{abstract}
Information on biodiversity and community structure is vital for monitoring the effects of climate change and other anthropogenic impacts. Benthic ecosystems of 5 sites off Viti Levu (Fiji), comprising 50 stations were sampled quantitatively revealing 13128 individuals of 230 species at a mean density of 273.5 ind. $\mathrm{m}^{-2}$. Common taxa included polychaetes (89 species), crustaceans (84 species), molluscs (50 species) and echinoderms ( 7 species). No species occurred in all 50 stations; the maximum distribution range was 45 stations occupied by the polychaete Aglaophamus sp. A total of 81 species $(35.2 \%)$ were restricted to single sites ('uniques'), highlighting spot endemism. Species richness and rarefaction curves provided high estimates of diversity. Multivariate analyses incorporating biological abundances and environmental factors showed 3 distinct clusters among sites characterising differences in benthic community structure. Strongest determinants of faunal distribution were depth, distance from reef and river, and sand content. The presence of heterogeneous faunal assemblages suggests the interplay of these factors at each site. Fauna in Nadi Bay (Shannon-Weiner diversity index $\left.H^{\prime}: 3.26\right)$, Suva Harbour $\left(H^{\prime}: 3.19\right)$ and Laucala Bay Lagoon $\left(H^{\prime}: 3.06\right)$ had high diversity indicative of biologically accommodated communities. Rewa River Estuary $\left(H^{\prime}: 2.42\right)$ and Nukubuco Reef drop-off $\left(H^{\prime}: 2.48\right)$ had low diversities, typical of habitats subjected to fluctuating environmental conditions. Benthic community structure in the lagoons around Viti Levu was rich and diverse. Biodiversity was greater than previously recorded from the Great Astrolabe Reef, Fiji (207 to 211 species) and Australia's Great Barrier Reef (154 species), but lower than in New Caledonia (311 species) and Tahiti (315 species).
\end{abstract}

KEY WORDS: Benthic communities $\cdot$ Marine biodiversity $\cdot$ Community structure $\cdot$ Spatial patterns Uniques $\cdot$ Spot endemism

Resale or republication not permitted without written consent of the publisher

\section{INTRODUCTION}

Fiji has one of the largest developed coral reef systems in the South Pacific (L. Zann 1992 unpubl. report), rated 6th in the world in terms of total coral area (Spalding et al. 2001). The 1000 or so reefs in Fiji include barrier, fringing and platform reefs, which are considered to be in good condition except those near the large urban centres, where pollution from sewage wastes, industries, poor land use and coastal development has impacted reefs (Nair 2003). The largest, most developed and populated of the Fiji Islands is Viti Levu, which is estimated to have approximately 150000 ha of coral reefs (WRI 1999).
Coral reefs support high biodiversity (Reaka-Kudla 1996, Gray 1997, Schlacher et al. 1998), while sedimentary habitats adjacent to reef structures in lagoons (Schlacher et al. 1998) are considered important in cross-habitat exchanges of material and energy. Various authors (Warwick \& Ruswahyuni 1987, Alongi 1990, Richer de Forges 1991, Newell \& Clavier 1997, Frouin \& Hutchings 2001) have highlighted the importance of tropical soft sediments in sheltering an abundant and diverse benthic fauna.

The 'Coral Triangle' is rich and diverse in marine life (WWF 2008) and within the Indo-Pacific covers all or parts of countries including Indonesia, Malaysia, Papua New Guinea and Philippines. Neighbouring 
countries (Fiji, Australia, New Caledonia) are also known to contain rich, but somewhat lower biodiversities. Roberts et al (2002) found that species richness peaked in the 'Coral Triangle' and fell eastwards across the Pacific. Although some ecological studies have been carried out on Fijian reef systems (WhippyMorris \& Pratt 1998, Coppard \& Campbell 2005, Zann 1992 unpubl. report) information on the country's benthic invertebrate biodiversity and distribution is minimal (Vuki et al. 2000). This lack of knowledge on invertebrate diversity and factors affecting community structure makes benthic ecological studies difficult, and diversity comparisons with neighbouring reef systems, islands and countries impossible. The need for baseline information for Fiji and knowledge of the ecological processes underpinning its benthic marine environments is crucial to the understanding and preservation of its biodiversity. Accordingly, we: (1) evaluated species richness and spatial pattern diversity within ecological communities in the lagoons around Viti Levu, (2) compared ecological communities among neighbouring lagoonal areas, (3) examined the interrelation between environmental variables and benthic community properties, and (4) compared diversity and community structure with similar habitats in the South Pacific.

Soft-bottom benthic communities have been used to investigate the effects of anthropogenic inputs and perturbations on coastal marine ecosystems (Pearson \& Rosenberg 1978, Keough \& Quinn 1991, Frouin 2000). Our study provides an overview of the community structure in lagoons around Viti Levu and will act as a baseline to help future monitoring and conservation programmes.

\section{MATERIALS AND METHODS}

Location and description. Fiji, a tropical archipelago, is located between latitudes $15^{\circ}$ and $22^{\circ} \mathrm{S}$, and between longitudes $177^{\circ}$ and $175^{\circ} \mathrm{E}$. Viti Levu (Fig. 1A) is by far the largest $\left(10388 \mathrm{~km}^{2}\right)$ and most populated island, with many of the major cities, industries and tourism facilities. Viti Levu is estimated to have about 150000 ha of coral reefs. This estimate is based on the total area of coral reefs in Fiji (an estimated 1 million ha, WRI 1999).

In order to distinguish between the different areas sampled and their associated communities, each area was assigned the term 'site' and a descriptive name was given to each based on location. Five sites were identified along the Viti Levu coastline. Sites 1 (Suva Harbour), 2 (Laucala Bay Lagoon), 3 (Rewa River Estuary) and 4 (Nukubuco Reef) were situated on the south/southeastern coastline, which is windward and consists of outer-shelf barrier reefs, with classic forereef, reef crest and back reef zones (Fig. 1B). Site 5 (Nadi Bay) was located on the northwestern coastline, which is on the island's leeward side and consists of mid- and inner-shelf platform reefs (Fig. 1C). A total of 50 stations were sampled from these 5 sites.

Site 1: Suva Harbour. Suva Harbour is a shallow, rectangular embayment, approximately $3 \mathrm{~km}$ long (northeast to southwest) and $2 \mathrm{~km}$ wide. The northeastern end of the harbour is shallow $(<30 \mathrm{~m})$. The harbour deepens seaward. To the southwest, the boundary of the harbour is marked by irregular bathymetric highs and channels. The floors of Suva Harbour and the inlets incised into the margins of the harbour are blanketed by thick, ubiquitous deposits of fine-grained organo-calcareous sediment (Shorten 1993).

Site 2: Laucala Bay Lagoon. Laucala Bay Lagoon is confined by Suva peninsula to the northwest, Rewa Delta to the northeast and Sosoikula and Nukubuco reefs to the south. The lagoon has 2 reef passages that allow access and interchange of water between lagoon and ocean. The water at the bottom of the lagoon moves northeastwards during high tides and southwards during ebb tides. The lagoon environment is mainly controlled by the Rewa River, which supplies high siliclastic sediment and dissolved chemical input (Schneider et al. 1995) along with a large supply of freshwater.

Site 3: Rewa River Estuary. The Rewa River is approximately $78000 \mathrm{~m}$ in length and drains approximately one-quarter of the entire Viti Levu land mass (predominantly southwards). Consequently high rates of sedimentation occur at the mouth of the river, contributing to increased turbidity as well as dissolved chemicals (pesticides, herbicides) and nutrients (Terry 1999).

Site 4: Nukubuco Reef drop-off. Nukubuco Reef is approximately $4.5 \mathrm{~km}$ long and nearly $2 \mathrm{~km}$ wide, with a large seagrass bed between the back reef and lagoon. On the seaward side of the reef crest lies the reef face, with rapidly deepening waters where high proportions of clay occur (Schneider et al. 1995).

Site 5: Nadi Bay. Nadi Bay is situated between the Mamanuca and Yasawa groups of islands and the mainland on the western (leeward) side of Viti Levu. The fringing reef in Nadi Bay does not enclose the harbour and a few patch reefs are situated sporadically within the bay. These formations allow unobstructed circulation of oceanic water, but provide no protection from the cyclone winds and waves.

Sampling procedure. A total of 200 samples was collected from the 50 stations using a $0.1 \mathrm{~m}^{2}$ Smith-McIntyre grab. At each station, 3 grab samples were taken for faunal analysis. Faunal samples were washed through a $1 \mathrm{~mm}$ sieve. Benthos retained on the sieve 

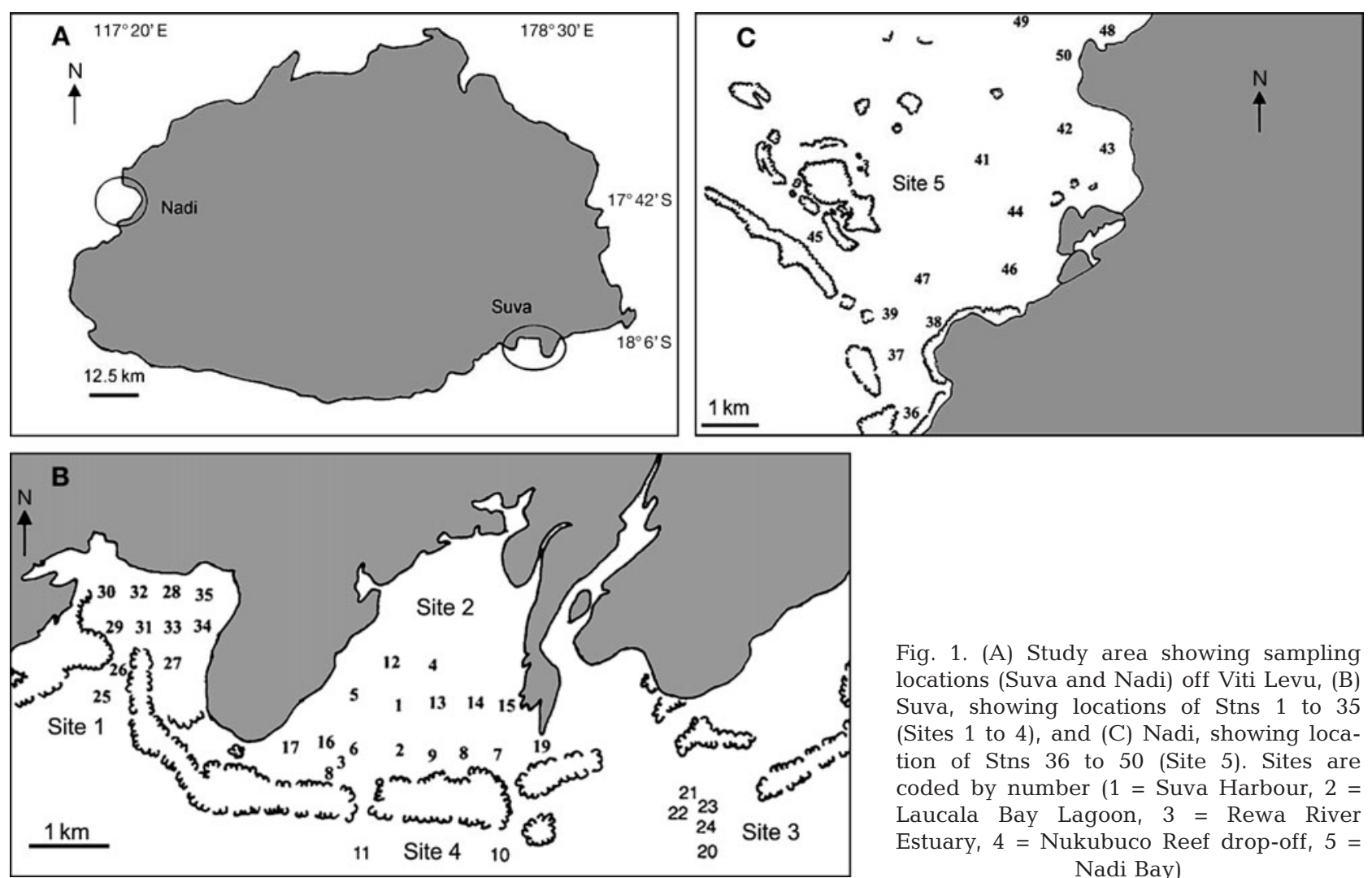

Fig. 1. (A) Study area showing sampling locations (Suva and Nadi) off Viti Levu, (B) Suva, showing locations of Stns 1 to 35 (Sites 1 to 4), and (C) Nadi, showing location of Stns 36 to 50 (Site 5). Sites are coded by number $(1=$ Suva Harbour, $2=$ Laucala Bay Lagoon, $3=$ Rewa River Estuary, 4 = Nukubuco Reef drop-off, 5 = Nadi Bay)

was fixed with $4 \%$ formaldehyde and stained with concentrated Rose Bengal. In the laboratory, the faunal samples were rinsed in tap water, the fauna picked out, sorted to major taxa and stored in $70 \%$ isopropanol. Identifications of benthos were carried out to the lowest taxonomic unit possible, followed by enumeration. Samples were deposited in the collections of Australian Museum (Australia), IRD (Institut de Recherche pour le Développement, New Caledonia) and Marine Studies Programme (Fiji).

A separate grab sample was collected at each station for sediment analysis. The sedimentary properties analysed included grain size, silt and clay fraction, organic matter content and carbonate/terrigenous material. Grain size fractions were obtained by dry sieving through a stack of sieves ranging from 2 to $0.063 \mathrm{~mm}$ pore sizes. The weight retained on each sieve was recorded as a percentage. Size analysis of naturally occurring fine sediments (silt/clay content) was achieved using the pipette method (Day 1965). This technique relies on the fact that particles in a dilute suspension settle through a column of water at velocities dependent upon their size (Folk 1974). Organic contents of the sediments were obtained by combustion of samples at high temperatures $\left(550^{\circ} \mathrm{C}\right)$. The weight change on combustion measures the organic content, and presence of carbonate and ter- rigenous materials present were calculated from the basaltic content of the sediments. Sediment was added to $4 \% \mathrm{HCl}$ then rinsed and washed through after $12 \mathrm{~h}$. The fraction of sediment that dissolved was classified as carbonate material, and the remaining fraction as terrigenous.

Data analysis. Univariate and multivariate analyses of data were performed using the PRIMER (Plymouth Routines in Multivariate Ecological Research) suite of programmes (Clarke \& Warwick 1994). Statistical analysis used was similar to that of Moyer et. al (2003).

Fauna data were entered into a square matrix (species $\times$ station) and a 4 th-root transformation was performed to lessen the weighting of the dominant species and increase the weighting of rare species. A logarithmic transformation was used to normalize relative abundance data. A triangular matrix of similarity coefficients was constructed from sample pairwise similarities (Bray-Curtis coefficient); the matrix was subjected to clustering and ordination analyses. Clustering was by a hierarchical agglomerative method resulting in a dendrogram, while ordination was by non-metric multidimensional scaling (MDS). In the 2-dimensional ordinations generated by MDS analysis, highly similar stations appear closer together than stations with lower rank similarities. 
Analyses of similarity (ANOSIM: Clarke \& Green 1988) were used for testing statisitical significance of sample groupings. The test statistic $R$ indicates some degree of discrimination between stations. When $R$ differs significantly from 0 and tends towards 1 , intra-site similarity is greater than inter-site similarity. SIMPER (non-metric similarity of percentages) analysis (Clarke \& Warwick 2001) was performed to calculate the degree of similarity between sites. Species contributing most to the similarity and dissimilarity of the groups were identified by this test.

Diversity indices, including Margalef's species richness index $(d)$, Pielou's evenness coefficient $\left(J^{\prime}\right)$, and the Shannon-Wiener diversity coefficient $\left(H^{\prime}\right)$, were calculated from pooled and untransformed faunal data sets. Although species richness is a natural measure of biodiversity, observed richness based on species counts over limited time periods often underestimates actual richness (Smith \& van Belle 1984). Species accumulation and rarefaction curves were calculated with the EstimateS 5 programme (Colwell 1999), which computes randomised species accumulation curves. The relationships between patterns in multivariate community structure and combinations of environmental variables were examined using the BIO-ENV procedure (Clarke \& Ainsworth 1993). This procedure calculates rank correlation between a similarity matrix derived from biotic data and matrices derived from various subsets of environmental variables, thereby defining suites of variables most closely correlated with the observed biotic structure. Range size (number of sites occupied by a single species) was calculated and species restricted to a single site were labelled 'uniques' (Schlacher et al. 1998).

\section{RESULTS}

\section{Community properties}

A total of 13128 individuals and 230 species (Table 1) were recorded, comprising 89 species of polychaetes $(38.7 \%), 84$ species of crustaceans $(36.5 \%), 50$ species of molluscs $(21.7 \%)$ and 7 species of echinoderms $(3.1 \%)$. Distributional ranges of soft sediment taxa were compressed. No single species occurred in all 50 stations (Fig. 2) and $20 \%$ of the species were restricted to 1 station. Conversely 81 species ( $35 \%$ ) were restricted to single sites ('uniques'), highlighting some degree of spot endemism in the community. The long 'tail' on the abundance-rank relationship suggests the presence of rare species (Fig. 3). Of these 81 'unique' species, $34.6 \%$ were molluscs, $32.1 \%$ polychaetes, $28.4 \%$ crustaceans and $4.9 \%$ were echinoderms. Cluster (Fig. 4A) and MDS (Fig. 4B) analysis of faunal data revealed 3
Table 1. List of species, total number of individuals collected and number of stations at which each species was found

\begin{tabular}{|c|c|c|}
\hline Species identified & $\begin{array}{c}\text { No. of ind. } \\
\text { collected }\end{array}$ & $\begin{array}{c}\text { No. of } \\
\text { stations }\end{array}$ \\
\hline Cossura sp. & 1724 & 43 \\
\hline Notomastus sp. 2 & 765 & 38 \\
\hline Aglaophamus sp. & 677 & 45 \\
\hline Ampelisca melanesiensis & 665 & 31 \\
\hline Cymadusa sp. & 492 & 32 \\
\hline Spionidae sp. 1 & 450 & 40 \\
\hline Piromis sp. & 438 & 39 \\
\hline Aricidea sp. & 423 & 39 \\
\hline Capitella sp. & 395 & 39 \\
\hline Notomastus sp. 1 & 309 & 37 \\
\hline Ampharetidae sp. & 267 & 38 \\
\hline Magelona sp. & 265 & 31 \\
\hline Sternaspis sp. & 251 & 27 \\
\hline Ancistrosyllis sp. & 243 & 37 \\
\hline Wildus parathambaroo & 214 & 21 \\
\hline Spionidae sp. 2 & 204 & 38 \\
\hline Paralacydonia sp. & 182 & 26 \\
\hline Mactridae sp. 1 & 170 & 22 \\
\hline Mediomastus sp. & 156 & 34 \\
\hline Scoloplos (S) sp. & 135 & 23 \\
\hline Photis pirloti & 130 & 18 \\
\hline Cerapus sp. & 120 & 3 \\
\hline Pilargis sp. & 119 & 28 \\
\hline Elasmopus sp. & 118 & 16 \\
\hline Horstileanira sp. & 112 & 34 \\
\hline Ostracoda sp. 3 & 111 & 12 \\
\hline Ostracoda sp. 1 & 109 & 14 \\
\hline Cumacea sp. 1 & 104 & 12 \\
\hline Tanaidacea sp. 1 & 98 & 13 \\
\hline Caulleriella sp. 2 & 89 & 23 \\
\hline Cirolana sp. 1 & 89 & 24 \\
\hline Cirriformia sp. & 89 & 25 \\
\hline Tellina sp. 2 & 87 & 26 \\
\hline Harpacticoida sp. & 83 & 19 \\
\hline Caulleriella sp.1 & 79 & 21 \\
\hline Tellina sp. 1 & 78 & 14 \\
\hline Leucothoe cf. diemenensis & 75 & 17 \\
\hline Scoloplos sp. & 75 & 22 \\
\hline Veneridae sp. 1 & 72 & 14 \\
\hline Terebellides sp. & 67 & 20 \\
\hline Mesochaetopterus sp. & 63 & 21 \\
\hline Thalassina sp. 1 & 63 & 21 \\
\hline Sabellidae & 62 & 12 \\
\hline Monoliropus sp. & 60 & 23 \\
\hline Glycera sp. 1 & 59 & 9 \\
\hline Lumbrinerides sp. & 58 & 17 \\
\hline Unknown sp. 1 & 55 & 7 \\
\hline Elaphognathia sp. 1 & 57 & 18 \\
\hline Spionidae sp. 5 & 54 & 9 \\
\hline Amphipholis squamata & 52 & 24 \\
\hline Ceratonereis sp. & 48 & 16 \\
\hline Schistomeringos sp. & 48 & 11 \\
\hline Spionidae sp. 4 & 46 & 14 \\
\hline Pagurus sp. 1 & 46 & 14 \\
\hline Tellina sp. 3 & 46 & 13 \\
\hline Scyphoproctus sp. & 45 & 14 \\
\hline Lucinidae & 44 & 18 \\
\hline Armandia sp. & 42 & 8 \\
\hline
\end{tabular}


Table 1 (continued)

\begin{tabular}{|c|c|c|c|c|c|}
\hline Species identified & $\begin{array}{l}\text { No. of ind. } \\
\text { collected }\end{array}$ & $\begin{array}{l}\text { No. of } \\
\text { station }\end{array}$ & Species identified & $\begin{array}{l}\text { No.of ind. } \\
\text { collected }\end{array}$ & $\begin{array}{l}\text { No. of } \\
\text { station }\end{array}$ \\
\hline Photis kepapa & 40 & 9 & Syllidae & 13 & 7 \\
\hline Loimia sp. & 39 & 10 & Tanaidacea sp. 2 & 13 & 6 \\
\hline Cirolana sp. 2 & 39 & 12 & Haplosyllis sp. & 11 & 7 \\
\hline Protella cf. similis & 39 & 9 & Bullidae sp. 2 & 11 & 7 \\
\hline Cardiidae sp. 4 & 38 & 11 & Mussels & 11 & 6 \\
\hline Macrophthalmus (Venitus) & & & Natica sp. 1 & 11 & 9 \\
\hline latreillei & 35 & 18 & Rissoidea & 11 & 5 \\
\hline Amphilocus sp. & 35 & 10 & Genetyllis sp. & 10 & 5 \\
\hline Ophiactis savignyi & 34 & 18 & Trypanosyllis sp. & 10 & 6 \\
\hline Maldanidae & 33 & 12 & Spionidae sp. 3 & 10 & 6 \\
\hline Glycera sp. 2 & 32 & 9 & Leander capensis & 10 & 6 \\
\hline Typhlocarcinops sp. 1 & 32 & 16 & Periclemenes sp. 1 & 10 & 5 \\
\hline Goniada sp. & 31 & 12 & Nassarius venustus & 10 & 4 \\
\hline Spionidae sp. 6 & 31 & 7 & Hesionella sp. & 9 & 7 \\
\hline Spionidae sp. 15 & 31 & 1 & Spionidae sp. 10 & 9 & 6 \\
\hline Cumacea sp. 2 & 31 & 6 & Hexapus sexpes & 9 & 5 \\
\hline Ostracoda sp. 2 & 32 & 12 & Typhlocarcinus stephensis & 9 & 6 \\
\hline Marphysa sp. & 28 & 10 & Thalamita sp. 1 & 8 & 4 \\
\hline Spionidae sp. 7 & 30 & 3 & Spionidae sp. 13 & 7 & 2 \\
\hline Processa sp. 1 & 28 & 12 & Arcania quinquespionsa & 7 & 4 \\
\hline Diogenes sp. 1 & 28 & 13 & Chloridina microphthalma & 7 & 3 \\
\hline Spionidae sp. 9 & 27 & 9 & Socarnopsis sp. & 7 & 4 \\
\hline Spionidae sp. 11 & 27 & 3 & Cominella sp. 1 & 7 & 4 \\
\hline Anthuridea & 26 & 8 & Turbo sp. 2 & 7 & 3 \\
\hline Macrophthalmus sp. 2 & 25 & 15 & Polyophthalmus sp. & 6 & 2 \\
\hline Paracaprella sp. & 25 & 10 & Unknown sp. 4 & 6 & 2 \\
\hline Polycirrus sp. & 24 & 7 & Hexapus sp. 2 & 6 & 5 \\
\hline Onuphis sp. & 23 & 2 & Amphithoe sp. & 6 & 2 \\
\hline Hexapus sp. 1 & 22 & 10 & Eusiridae & 6 & 4 \\
\hline Macrophthalmus sp. 1 & 22 & 11 & Cardiidae sp. 1 & 6 & 3 \\
\hline Macrophthalmus dentatus & 22 & 11 & Polynoidea sp. 2 & 5 & 6 \\
\hline Laonome sp. & 21 & 10 & Spionidae sp. 12 & 5 & 5 \\
\hline Polynoidea sp.1 & 21 & 6 & Thalamita sexlobata & 5 & 2 \\
\hline Anadara sp. 1 & 21 & 8 & Portunus (Xiphonectus) & & \\
\hline Sabellastarte sp. & 20 & 8 & hastatoides & 5 & 4 \\
\hline Typhlocarcinus nudus & 20 & 12 & Portunus sp. 1 & 5 & 4 \\
\hline Gitana sp. & 20 & 5 & Oratosquillina gravieri & 5 & 2 \\
\hline Bulla sp. 1 & 19 & 9 & Cyclopoida sp. & 5 & 3 \\
\hline Nassarius concinnus & 19 & 10 & Cardiidae sp. 2 & 5 & 3 \\
\hline Harmothoe sp. & 18 & 10 & Cerithium sp. & 5 & 3 \\
\hline Phyllodocidae & 18 & 11 & Turritella sp. 1 & 5 & 3 \\
\hline Phyllodoce sp. & 18 & 15 & Vexillum sp. 1 & 5 & 2 \\
\hline Pseudeurythoe sp. & 17 & 6 & Turridae sp. 1 & 5 & 5 \\
\hline Poecilochaetus sp. & 17 & 8 & Arabella sp. & 4 & 3 \\
\hline Athanas nitescens & 17 & 8 & Serpulidae & 4 & 3 \\
\hline Cumacea sp. 3 & 17 & 6 & Spionidae sp. 8 & 4 & 1 \\
\hline Pseudoprotomima sp. & 17 & 7 & Unknown sp. 6 & 4 & 1 \\
\hline Leptosquilla schmeltzii & 16 & 8 & Lybistes paucidentus & 4 & 3 \\
\hline Leucothoe gaviata & 16 & 8 & Podophthalmus vigil & 4 & 3 \\
\hline Ophionereis variegate & 16 & 11 & Tetrias fisheri & 4 & 4 \\
\hline Leptochela sp. 1 & 15 & 6 & Parapasiphae sp. 1 & 4 & 3 \\
\hline Alpheus sp. 1 & 15 & 8 & Callianassa sp. 1 & 4 & 1 \\
\hline Alpheus longichaelis & 15 & 8 & Podoceruscrenulates & 4 & 2 \\
\hline Alpheus chacei & 15 & 6 & Mitre sp. 1 & 4 & 3 \\
\hline Parambasia cf. nui & 15 & 5 & Mitre sp. 2 & 4 & 1 \\
\hline Syllis sp. 1 & 14 & 5 & Turbo sp. 1 & 4 & 3 \\
\hline Syllis sp. 2 & 14 & 6 & Eurythoe sp. & 3 & 2 \\
\hline Unknown sp. 5 & 14 & 4 & Owenia sp. & 3 & 1 \\
\hline Typhlocarcinus sp. 1 & 14 & 16 & Scalibregmidae & 3 & 3 \\
\hline
\end{tabular}


Table 1 (continued)

\begin{tabular}{|c|c|c|}
\hline Species identified & $\begin{array}{l}\text { No. of ind. } \\
\text { collected }\end{array}$ & $\begin{array}{l}\text { No. of } \\
\text { station }\end{array}$ \\
\hline Spionidae sp. 21 & 3 & 3 \\
\hline Serolia sp. 2 & 3 & 1 \\
\hline Gitanopsis sp. & 3 & 2 \\
\hline Cardiidae sp. 3 & 3 & 2 \\
\hline Marginella sp. 1 & 3 & 3 \\
\hline Myacea sp. 1 & 3 & 2 \\
\hline Natica sp. 2 & 3 & 3 \\
\hline Nematonereis sp. & 2 & 2 \\
\hline Spionidae sp. 14 & 2 & 1 \\
\hline Spionidae sp. 18 & 2 & 2 \\
\hline Spionidae sp. 22 & 2 & 2 \\
\hline Unknown sp. 2 & 2 & 2 \\
\hline Unknown sp. 3 & 2 & 1 \\
\hline Portunus sp. 2 & 2 & 2 \\
\hline Paranamixis sp. & 2 & 1 \\
\hline Nebalia sp. & 2 & 1 \\
\hline Arcidae sp. 1 & 2 & 2 \\
\hline Arcidae sp. 2 & 2 & 2 \\
\hline Atys sp. 2 & 2 & 1 \\
\hline Bullidae sp. 3 & 2 & 1 \\
\hline Olividae sp. 2 & 2 & 2 \\
\hline Tellina sp. 4 & 2 & 1 \\
\hline Pectinidae sp. 1 & 2 & 1 \\
\hline Mytilidae sp. 1 & 2 & 1 \\
\hline Epitonidae sp. & 2 & 1 \\
\hline Maretia planulata & 2 & 1 \\
\hline Streptosyllis sp. & 1 & 3 \\
\hline Spionidae sp. 16 & 1 & 1 \\
\hline Spionidae sp. 17 & 1 & 1 \\
\hline Spionidae sp. 19 & 1 & 1 \\
\hline Spionidae sp. 20 & 1 & 1 \\
\hline Spionidae sp. 23 & 1 & 1 \\
\hline Iphiculus spongiosus & 1 & 1 \\
\hline Nursilia sp. & 1 & 1 \\
\hline \multicolumn{3}{|l|}{ Parthenope (Rhino- } \\
\hline lambrus) sp. 1 & 1 & 1 \\
\hline Thalamita sp. 2 & 1 & 1 \\
\hline Thalamita spinifera & 1 & 1 \\
\hline Crangon sp. 1 & 1 & 1 \\
\hline Galathea sp. 1 & 1 & 1 \\
\hline Serolia sp. 1 & 1 & 1 \\
\hline Isopoda sp. & 1 & 1 \\
\hline Sabidae sp. & 1 & 1 \\
\hline Acteon sp. 1 & 1 & 1 \\
\hline Atys sp. 1 & 1 & 1 \\
\hline Collumbellidae sp. 1 & 1 & 1 \\
\hline Fasciolaridae sp. 1 & 1 & 1 \\
\hline Fasciolaridae sp. 2 & 1 & 1 \\
\hline Nassarius sp. 1 & 1 & 1 \\
\hline Pectinidae sp. 2 & 1 & 1 \\
\hline Pyramidellidae sp. 1 & 1 & 1 \\
\hline Pyramidellidae sp. 2 & 1 & 1 \\
\hline Solecurtidae & 1 & 1 \\
\hline Cardiidae sp. 5 & 1 & 1 \\
\hline Clypeaster reticulatus & 1 & 1 \\
\hline Fibularia ovulum & 1 & 1 \\
\hline Echinoneus cyclostomus & 1 & 1 \\
\hline
\end{tabular}

distinct groupings. Sites 1 and 2 formed one cluster, Site 3 formed a second cluster and a third cluster comprised Sites 4 and 5. Principal component analysis (PCA) ordination of environmental variables superimposed on faunal data also showed 3 major clusters. Sites 1, 2 and 3 form one cluster, Site 4 forms a second cluster and Site 5 forms a third cluster (Fig. 5). A mean density of 273.5 ind. $\mathrm{m}^{-2}$ was recorded. Mean densities were similar in Sites 1 and 2 (609 to $781 \mathrm{~m}^{-2}$ ), high in Sites 3 and 5 (1040.8 to $\left.1548.6 \mathrm{~m}^{-2}\right)$ and lowest in Site 4 $\left(226.6 \mathrm{~m}^{-2}\right)$. Average values for the taxa, numbers of species and individuals are presented in Table 2. Mean species diversity $H^{\prime}$ varied from 2.41 to 3.26. Species causing similarities and dissimilarities within and between sites are shown in Table 3.

Sampling effort was taken into account when comparing species richness. Differences in the richness and relative abundances of species in the communities sampled are shown by differences in the shapes of the species accumulation and rarefaction curves. Since the number of species in any community is finite, a species accumulation curve that reaches an asymptote indicates that no additional species are to be found. Projections from the species accumulation curves (Fig. 6) all extrapolate the total richness at the study site to over 250 species: 251 (Bootstrap), 277 (Jackknife 1) and 302 (Jackknife 2). Accumulation (Fig. 7) and rarefaction curves (Fig. 8) do not reach asymptotes, suggesting inadequate sampling. The more concave-downward the curve, the better sampled the community, confirming that Site 4 (Nukubuco Reef) was the most under-sampled.

\section{Site 1: Suva Harbour}

A total of 2010 individuals and 113 species at a mean density of 609 ind. $\mathrm{m}^{-2}$ were recorded in Site 1 (Table 2). Of the taxa collected, 14 (17.3\%) were 'uniues'. Polychaetes were the dominant group comprising $49 \%$ (55) of the population, followed by crustaceans $(31 \%, 35)$, molluscs $(17 \%, 19)$ and echinoderms $(3 \%, 4)$ (Table 4). Highest species counts were recorded at stations in close proximity to the shore and within the channel. Lowest abundances were recorded at stations outside the passages. Diversity measures in Site 1 (Table 5) showed that Stn 25 had the lowest species richness $(d)$, while Stn 26 had the highest. Species diversity $\left(H^{\prime}\right)$ was lowest at Stn 34 and greatest at Stn 26. Mean species diversity was 3.19. The ANOSIM of Site 1 (Table 6) showed no significant differences between Sites 2 and $5(R=0.242$, $\mathrm{p}=0.3 \%$ and $R=0.434, \mathrm{p}=0.1 \%$ respectively), but did not show significant difference between Sites 3 and $4(R=0.565, \mathrm{p}=0.2 \%$ and $R=0.828, \mathrm{p}=1.3 \%$, 


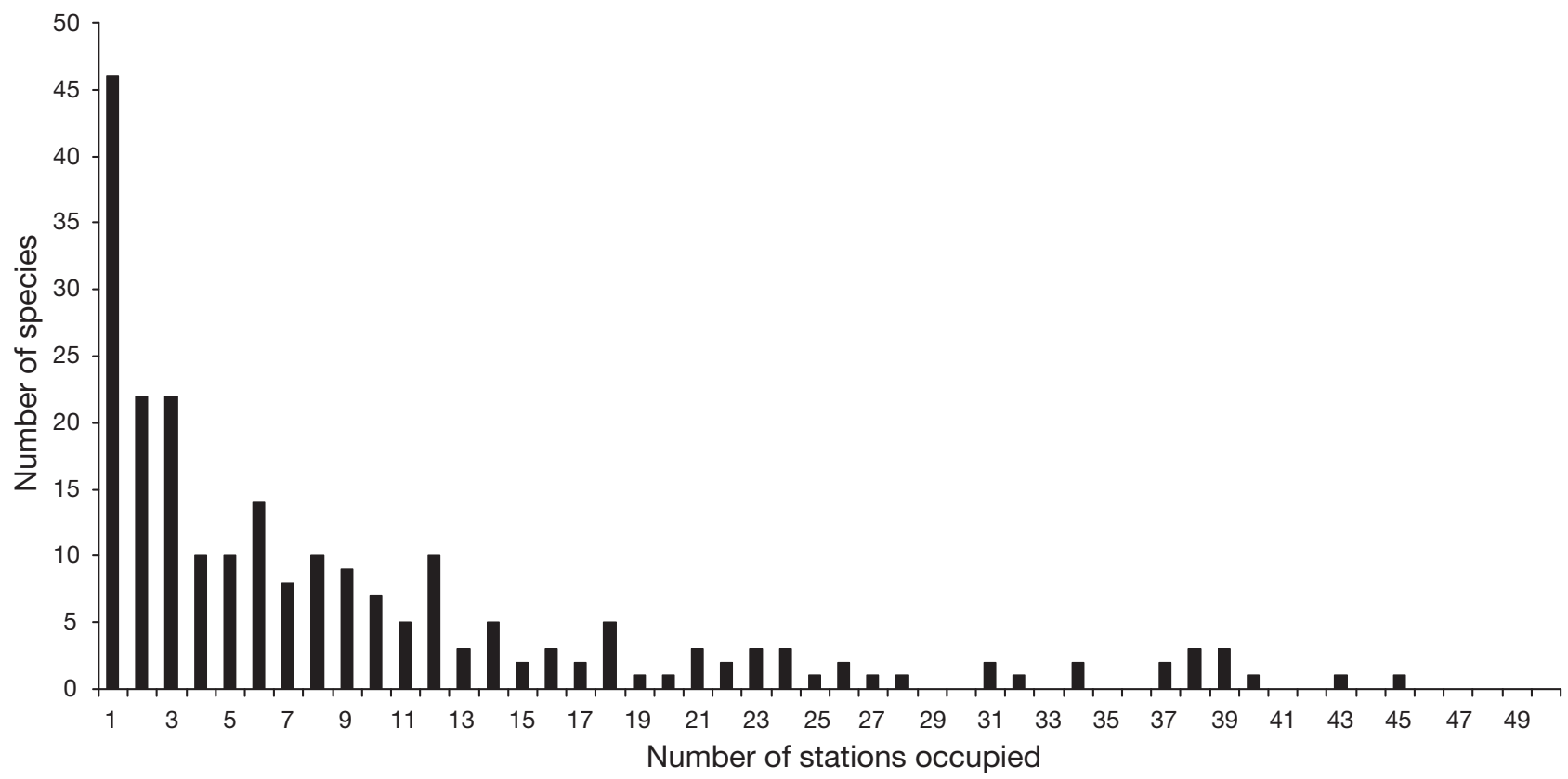

Fig. 2. Frequency distribution of species occurrences across stations. Range size is the number of stations occupied by a species out of a total of 50 stations

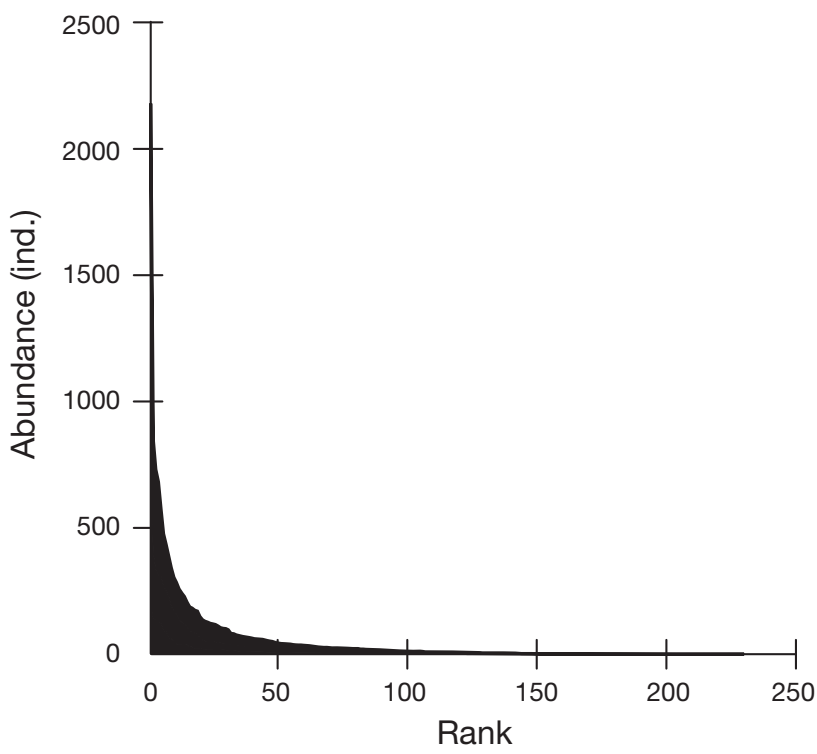

Fig. 3. Rank-abundance plot (log abundance [no. of individuals] against rank [species sequence]). Plot based on 230 species that are ranked so that the most common species are on the left

respectively). SIMPER analysis showed that at Site 1 the polychaetes Aglaophamus sp. and Paralacydonia sp. contributed most to within group similarity (16.9 and $10.6 \%$ respectively), along with Piromis sp.(9.9\%), Spionidae sp. 1 (7.8\%) and Notomastus sp. 2 (7.2\%, Table 3$)$.
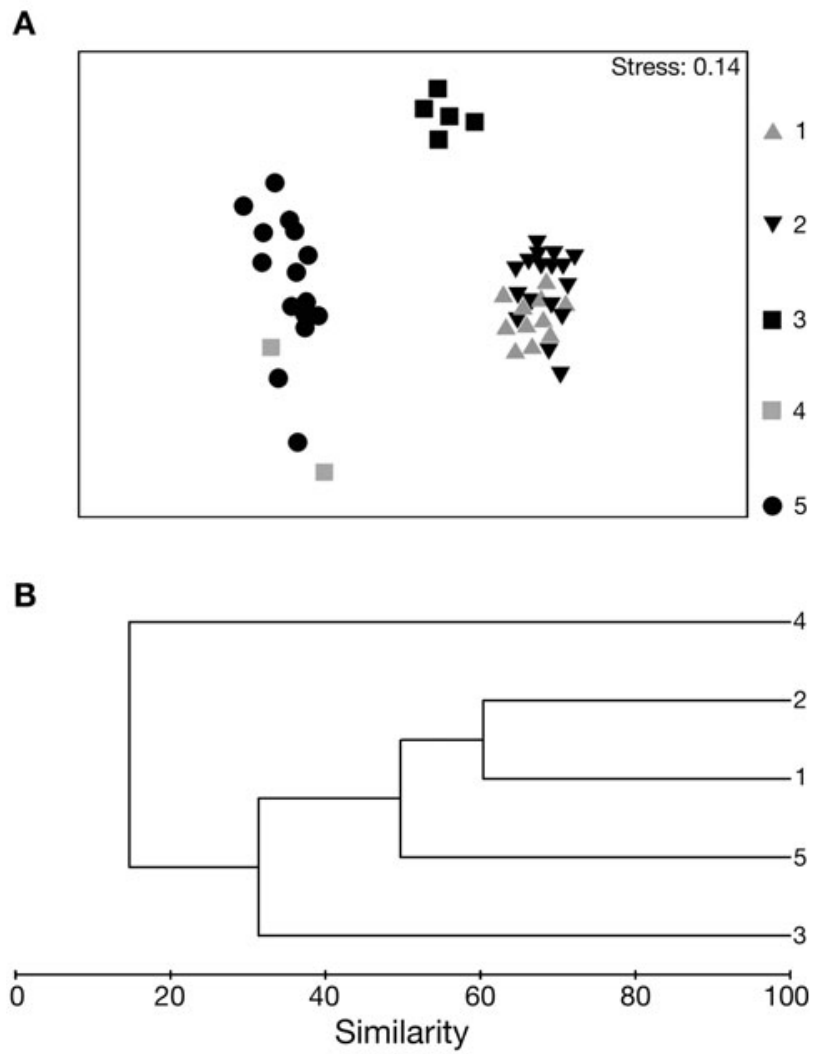

Fig. 4. (A) MDS plot and (B) dendrogram of all sites based on the Bray-Curtis similarity index. Sites are coded 1 to 5 (see Fig. 1) 


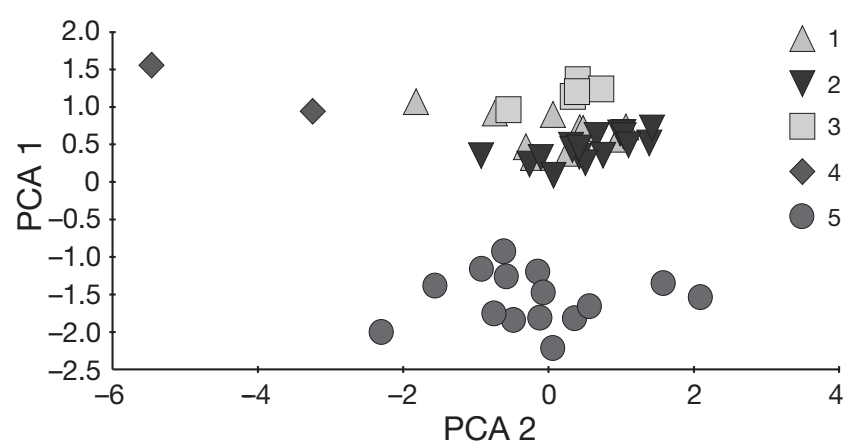

Fig. 5. PCA (principal component analysis) ordination of faunal data superimposed with environmental factors (depth, distance from reef and river, sand content) that contribute most to similarities in community structure between sites. Sites are coded 1 to 5 (see Fig. 1)

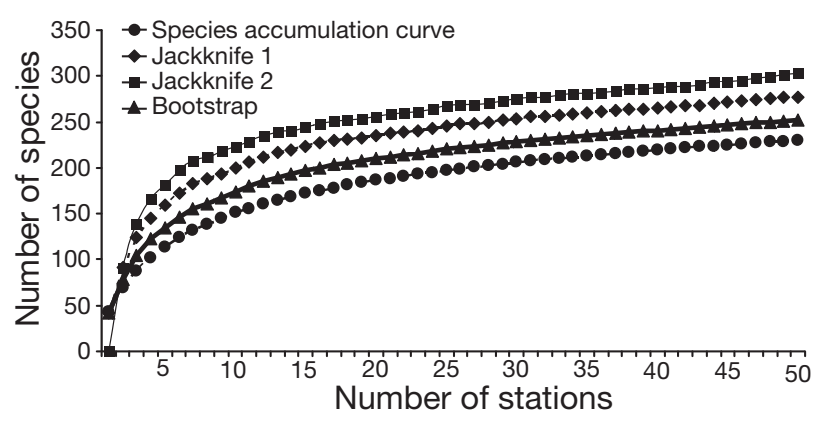

Fig. 6. Species accumulation curves based on EstimateS 5 (Colwell 1999)
Site 2: Laucala Bay Lagoon

A total of 3985 individuals belonging to 138 species at a mean density of 781 ind. $\mathrm{m}^{-2}$ was recorded in Site 2 (Table 2). Nineteen (23.5\%) 'uniques' were recorded in this site. Crustaceans were the dominant group comprising $46 \%$ (63) of the population, followed by polychaetes $(40 \%, 55)$, molluscs $(12 \%, 17)$ and echinoderms $(2 \%, 3)$ (Table 4). Stations closer to the shore had greater abundances than those further from shore. Lowest abundance was recorded in the middle of the lagoon. Stn 1 had the lowest species richness $(d)$, while Stn 17 had the highest. Lowest species diversity $\left(H^{\prime}\right)$ was recorded at Stn 14 and greatest at Stn 17. Mean species diversity $\left(H^{\prime}\right)$ was 3.06 . The ANOSIM of Site 2 (Table 6) showed no significant differences from Sites 1,3 and $5(R=0.242, \mathrm{p}=0.3 \%$; $R=$ $0.159, \mathrm{p}=17.1 \%$ and $R=0.434, \mathrm{p}=0.1 \%$, respectively), but did show a significant difference from Site 4 ( $R=$ $0.828, p=1.3 \%$ ). SIMPER analysis (Table 3 ) indicated that at Site 2 the polychaetes Notomastus sp. 2 and Piromis sp. made the largest contribution to the group similarities (11.4 and 10.1\%, respectively), followed by Spionidae sp. 1 (8.4\%), Aricidea sp. (8.3\%), Ampelisca melanesiensis (7.2\%) and Notomastus sp. 1 (6.1\%).

\section{Site 3: Rewa River Estuary}

Seventy species and 2323 individuals were recorded at Site 3 (Table 2). The mean density was 1548.6 ind. $\mathrm{m}^{-2}$. Polychaetes were the dominant taxa $(55 \%, 39)$,

Table 2. Principal environmental and biological characteristics of the 5 sites (mean values for each site and for all stations). See Fig. 1 for sites; $n=$ no. of stations

\begin{tabular}{|c|c|c|c|c|c|c|}
\hline & & & Site & & & All sites \\
\hline & $\begin{array}{c}1 \\
(\mathrm{n}=11)\end{array}$ & $\begin{array}{c}2 \\
(\mathrm{n}=17)\end{array}$ & $\begin{array}{c}3 \\
(n=5)\end{array}$ & $\begin{array}{c}4 \\
(\mathrm{n}=2)\end{array}$ & $\begin{array}{c}5 \\
(\mathrm{n}=15)\end{array}$ & $(\mathrm{n}=50)$ \\
\hline Environmental parameters & & & & & & \\
\hline Depth (m) & 45.4 & 18.9 & 29.4 & 168 & 22.5 & 31.08 \\
\hline Percent gravel (>1 mm) & 6.29 & 1.49 & 0.58 & 57.3 & 6.87 & 14.51 \\
\hline Percent sand $(0.125-1 \mathrm{~mm})$ & 31.89 & 29.74 & 6.90 & 35.30 & 38.19 & 28.40 \\
\hline Percent silt $(0.63 \mu \mathrm{m})$ & 43.29 & 50.18 & 79.72 & 6.17 & 46.26 & 45.12 \\
\hline Percent clay $(<0.63 \mu \mathrm{m})$ & 12.29 & 18.50 & 21.89 & 1.15 & 9.09 & 12.58 \\
\hline Organic content $(\%)$ & 11.18 & 14.88 & 10.14 & 6.70 & 7.37 & 10.05 \\
\hline Biological parameters & & & & & & \\
\hline No. ind. $\mathrm{m}^{-2}$ & 609 & 781 & 1548.6 & 226.6 & 1040.8 & 273.5 \\
\hline No. of species & 42.27 & 49.17 & 36.4 & 16 & 49.46 & 44.66 \\
\hline Species diversity $\left(H^{\prime}\right)$ & 3.19 & 3.06 & 2.41 & 2.48 & 3.26 & 3.06 \\
\hline Evenness $\left(J^{\prime}\right)$ & 0.86 & 0.82 & 0.66 & 0.93 & 0.88 & 0.84 \\
\hline No. of polychaetes & 5.0 & 3.23 & 7.6 & 8.5 & 4.47 & 1.78 \\
\hline No. of crustaceans & 3.18 & 3.71 & 4.2 & 2.0 & 4.2 & 1.68 \\
\hline No. of molluscs & 1.82 & 1.0 & 1.6 & 0 & 2.4 & 1.0 \\
\hline No. of echinoderms & 0.27 & 0.17 & 0.6 & 0 & 0.33 & 0.14 \\
\hline
\end{tabular}


Table 3. Species causing similarities within groups and dissimilarities between groups (Bray Curtis similarity indices). Species that contribute towards $50 \%$ of cumulative data (Cum. \%) are listed in descending order of their percent contributions (Cont. \%). Average similarities and dissimilarities (\%) are given in parentheses. See Fig. 1 for site locations

\begin{tabular}{|c|c|c|c|c|c|c|}
\hline Species & Cont. (\%) & Cum. $(\%)$ & Site & Species & Cont. (\%) & Cum. $(\%)$ \\
\hline \multirow{2}{*}{\multicolumn{3}{|c|}{$\begin{array}{l}\text { 1: Suva Harbour } \\
\text { (Average similarity: 42.56) }\end{array}$}} & & Notomastus sp. 2 & 4.63 & 40.88 \\
\hline & & & & Capitella sp. & 4.27 & 45.15 \\
\hline Aglaophamus sp. & 16.99 & 16.99 & & Aricidea sp. & 3.67 & 48.82 \\
\hline Paralacydonia sp. & 10.65 & 27.64 & & Paralacydonia sp. & 2.80 & 51.62 \\
\hline Piromis sp. & 9.95 & 37.59 & & & & \\
\hline Spionidae sp. 1 & 7.78 & 45.37 & \multicolumn{4}{|c|}{2 \& 3: Laucala Bay \& Rewa River } \\
\hline \multirow{2}{*}{ Notomastus sp. 2} & 7.17 & 52.54 & \multicolumn{3}{|c|}{ (Average dissimilarity = 74.27) } & \\
\hline & & & & Cossura sp. 0 & 29.46 & 29.46 \\
\hline \multirow{2}{*}{\multicolumn{3}{|c|}{$\begin{array}{l}\text { 2: Laucala Bay } \\
\text { (Average similarity: } 32.97 \text { ) }\end{array}$}} & & Notomastus sp. 2 & 6.60 & 36.06 \\
\hline & & & & Cerapus sp. & 5.81 & 41.87 \\
\hline Notomastus sp. 2 & 11.36 & 11.36 & & Capitella sp. & 3.58 & 45.45 \\
\hline Piromis sp. & 10.08 & 21.44 & & Aricidea sp. & 3.57 & 49.02 \\
\hline Spionidae sp. 1 & 8.44 & 29.87 & & Aglaophamus sp. & 3.08 & 52.10 \\
\hline Aricidea sp. & 8.29 & 38.17 & & & & \\
\hline Ampelisca melanesiensis & 7.20 & 45.37 & $2 \& 4$ & : Laucala Bay \& Fore-Reef & & \\
\hline Notomastus sp. 1 & 6.14 & 51.51 & (Ave & age dissimilarity $=78.57$ ) & & \\
\hline \multicolumn{3}{|l|}{ 3: Rewa River } & & Aglaophamus sp & 6.51 & 6.51 \\
\hline \multicolumn{3}{|l|}{ (Average similarity: 34.40 ) } & & Paralacydonia sp. & 5.91 & 12.43 \\
\hline Cossura sp. & 27.81 & 27.81 & & Piromis sp. & 5.56 & 17.98 \\
\hline Capitella sp. & 11.74 & 39.55 & & Spionidae sp. 1 & 5.12 & 23.10 \\
\hline Aglaophamus sp. & 11.20 & 50.74 & & Ampelisca melanesiensis & 4.67 & 27.77 \\
\hline & & & & Cossura sp. & 4.31 & 32.08 \\
\hline \multicolumn{3}{|l|}{ 4: Fore-Reef } & & Wildus parathambaroo & 4.11 & 36.19 \\
\hline \multicolumn{3}{|l|}{ (Average similarity: 47.06) } & & Aricidea sp. & 4.05 & 40.23 \\
\hline Wildus parathambaroo & 21.88 & 21.88 & & Ampharetidae sp. & 2.79 & 43.03 \\
\hline Ampelisca melanesiensis & 21.88 & 43.75 & & Spionidae sp. 2 & 2.52 & 45.55 \\
\hline Notomastus sp. 2 & 15.63 & 59.38 & & Anthuridea & 2.38 & 47.93 \\
\hline \multirow{2}{*}{\multicolumn{7}{|c|}{ 5: Nadi Bay }} \\
\hline & & & & & & \\
\hline Cymadusa sp. & 13.31 & 13.31 & \multicolumn{4}{|c|}{$\begin{array}{l}1 \text { \& 4: Suva Harbour \& Fore-Reef } \\
\text { (Average dissimilarity }=77.57 \text { ) }\end{array}$} \\
\hline Horstileanira sp. & 8.51 & 21.83 & & Notomastus sp. 2 & 10.09 & 10.09 \\
\hline Magelona sp. & 7.58 & 29.41 & & Ampelisca melanesiensis & 6.21 & 16.30 \\
\hline Spionidae sp. 1 & 6.16 & 35.57 & & Piromis sp. & 5.15 & 21.45 \\
\hline Spionidae sp. 2 & 5.38 & 40.95 & & Spionidae sp. 1 & 4.68 & 26.13 \\
\hline Ampelisca melanesiensis & 4.45 & 45.40 & & Aricidea sp. & 4.19 & 30.32 \\
\hline Thalassina sp. 1 & 4.30 & 49.70 & & Notomastus sp. 1 & 4.14 & 34.46 \\
\hline Sternaspis sp. & 3.58 & 53.28 & & Capitella sp. & 3.78 & 38.25 \\
\hline \multirow{2}{*}{\multicolumn{3}{|c|}{$\begin{array}{l}1 \text { \& 2: Suva Harbour \& Laucala Bay } \\
\text { (Average dissimilarity }=69.47 \text { ) }\end{array}$}} & & Wildus parathambaroo & 3.42 & 41.67 \\
\hline & & & & Cossura sp. & 2.92 & 44.59 \\
\hline Notomastus sp. 2 & 8.31 & 8.31 & & Cymadusa sp. & 2.84 & 47.44 \\
\hline Ampelisca melanesiensis & 5.06 & 13.37 & & Ancistrosyllis sp. & 2.39 & 49.83 \\
\hline Aglaophamus sp. & 4.72 & 18.09 & & Anthuridea & 2.07 & 51.89 \\
\hline Paralacydonia sp. & 4.31 & 22.40 & & & & \\
\hline Cossura sp. & 4.24 & 26.63 & \multirow{2}{*}{\multicolumn{4}{|c|}{$\begin{array}{l}3 \text { \& 4: Rewa River \& Fore-Reef } \\
\text { (Average dissimilarity }=86.65 \text { ) }\end{array}$}} \\
\hline Spionidae sp. 1 & 4.12 & 30.76 & & & & \\
\hline Notomastus sp. 1 & 3.09 & 33.85 & & Cossura sp. & 32.16 & 32.16 \\
\hline Capitella sp. & 2.92 & 36.76 & & Cerapus sp. & 6.90 & 39.06 \\
\hline Piromis sp. & 2.83 & 39.60 & & Capitella sp. & 5.75 & 44.81 \\
\hline Aricidea sp. & 2.83 & 42.42 & & Notomastus sp. 2 & 5.35 & 50.16 \\
\hline Cymadusa sp. & 2.32 & 44.74 & & & & \\
\hline Ampharetidae sp. & 2.12 & 46.86 & $1 \& 5$ & : Suva Harbour \& Nadi Bay & & \\
\hline Spionidae sp. 2 & 2.04 & 48.90 & (Ave & age dissimilarity = 80.80) & & \\
\hline Ancistrosyllis sp. & 2.00 & 50.90 & & Aglaophamus sp. & 6.33 & 6.33 \\
\hline & & & & Cymadusa sp. & 4.37 & 10.69 \\
\hline \multicolumn{3}{|l|}{1 \& 3: Suva Harbour \& Rewa River } & & Cossura sp. & 4.30 & 15.00 \\
\hline \multicolumn{3}{|l|}{ (Average dissimilarity = 75.73) } & & Paralacydonia sp. & 4.24 & 19.24 \\
\hline Cossura sp. & 30.14 & 30.14 & & Ampelisca melanesiensis & 3.61 & 22.85 \\
\hline Cerapus sp. & 6.11 & 36.25 & & Piromis sp. & 3.58 & 26.42 \\
\hline
\end{tabular}


Table 3 (continued)

\begin{tabular}{|c|c|c|c|}
\hline Site & Species & Cont. (\%) & Cum. $(\%)$ \\
\hline & Spionidae sp. 1 & 3.56 & 29.99 \\
\hline & Aricidea sp. & 2.80 & 32.78 \\
\hline & Magelona sp. & 2.52 & 35.31 \\
\hline & Notomastus sp. 2 & 2.46 & 37.77 \\
\hline & Sternaspis sp. & 2.34 & 40.10 \\
\hline & Ampharetidae sp. & 2.29 & 42.39 \\
\hline & Thalassina sp. 1 & 1.66 & 44.05 \\
\hline & Spionidae sp. 2 & 1.63 & 45.68 \\
\hline & Mactridae sp. 1 & 1.56 & 47.25 \\
\hline & Horstileanira sp. & 1.53 & 48.78 \\
\hline & Capitella sp. & 1.36 & 50.14 \\
\hline \multicolumn{4}{|c|}{$\begin{array}{l}2 \text { \& 5: Laucala Bay \& Nadi Bay } \\
\text { (Average dissimilarity = 78.69) }\end{array}$} \\
\hline & Notomastus sp. 2 & 7.42 & 7.42 \\
\hline & Ampelisca melanesiensis & 5.66 & 13.08 \\
\hline & Cymadusa sp. & 4.20 & 17.27 \\
\hline & Cossura sp. & 3.52 & 20.80 \\
\hline & Piromis sp. & 3.36 & 24.16 \\
\hline & Spionidae sp. 1 & 3.08 & 27.24 \\
\hline & Aglaophamus sp. & 3.06 & 30.30 \\
\hline & Aricidea sp. & 3.02 & 33.32 \\
\hline & Notomastus sp. 1 & 2.90 & 36.22 \\
\hline & Capitella sp. & 2.69 & 38.92 \\
\hline & Magelona sp. & 2.63 & 41.54 \\
\hline & Wildus parathambaroo & 2.08 & 43.63 \\
\hline & Ampharetidae sp. & 2.01 & 45.64 \\
\hline & Sternaspis sp. & 1.88 & 47.52 \\
\hline & Ancistrosyllis sp. & 1.82 & 49.35 \\
\hline & Thalassina sp. 1 & 1.58 & 50.93 \\
\hline \multicolumn{4}{|c|}{$\begin{array}{l}\text { 3 \& 5: Rewa River \& Nadi Bay } \\
\text { (Average dissimilarity = 83.96) }\end{array}$} \\
\hline & Cossura sp. & 26.67 & 26.67 \\
\hline & Cerapus sp. & 5.27 & 31.93 \\
\hline & Notomastus sp. 2 & 4.53 & 36.46 \\
\hline & Aglaophamus sp. & 4.24 & 40.70 \\
\hline & Capitella sp. & 3.74 & 44.44 \\
\hline & Aricidea sp. & 3.00 & 47.44 \\
\hline & Cymadusa sp. & 2.84 & 50.28 \\
\hline \multicolumn{4}{|c|}{$\begin{array}{l}4 \text { \& 5: Fore-Reef } \& \text { Nadi Bay } \\
\text { (Average dissimilarity }=84.86 \text { ) }\end{array}$} \\
\hline & Cymadusa sp. & 6.03 & 6.03 \\
\hline & Ampelisca melanesiensis & 6.01 & 12.04 \\
\hline & Magelona sp. & 3.48 & 15.52 \\
\hline & Wildus parathambaroo & 3.48 & 19.00 \\
\hline & Spionidae sp. 1 & 3.26 & 22.26 \\
\hline & Aglaophamus sp. & 3.22 & 25.49 \\
\hline & Cossura sp. & 3.12 & 28.61 \\
\hline & Horstileanira sp. & 2.64 & 31.25 \\
\hline & Sternaspis sp. & 2.60 & 33.85 \\
\hline & Thalassina sp. 1 & 2.54 & 36.39 \\
\hline & Notomastus sp. 2 & 2.53 & 38.92 \\
\hline & Anthuridea & 2.28 & 41.20 \\
\hline & Spionidae sp. 2 & 2.02 & 43.22 \\
\hline & Ampharetidae sp. & 1.92 & 45.14 \\
\hline & Tanaidacea sp. 1 & 1.63 & 46.77 \\
\hline & Capitella sp. & 1.58 & 48.35 \\
\hline & Elasmopus sp. & 1.57 & 49.92 \\
\hline & Piromis sp. & 1.57 & 51.49 \\
\hline
\end{tabular}

followed by crustaceans $(30 \%, 21)$, molluscs $(11 \%, 7)$ and echinoderms $(4 \%, 3)$. Of the species collected, $7.4 \%$ were 'uniques'. Low species counts were recorded at this site. Stn 23 had the lowest species richness $(d)$ and species diversity $\left(H^{\prime}\right)$, and Stn 20 had the highest $d$ and $H^{\prime}$ (Table 5). Mean $H^{\prime}$ was 2.41. The ANOSIM of Site 3 (Table 6) showed significant difference from Sites 1 and $4(R=0.565, \mathrm{p}=0.2 \%$ and $R=$ $0.691, \mathrm{p}=4.8 \%$ ), but no significant differences from Sites 2 and $5(R=0.645, \mathrm{p}=2.9 \%$ and $R=0.181, \mathrm{p}=$ $9.6 \%$, respectively). SIMPER analysis showed that at Site 3 the polychaetes Cossura sp., Capitella sp. and Aglaophamus sp. made the largest contribution to group similarity $(27.8,11.7$ and $11.2 \%$, respectively, Table 3).

\section{Site 4: Nukubuco Reef drop-off}

A total of 21 species and 136 individuals were recorded at Site 4 . The mean density was 226.6 ind. $\mathrm{m}^{-2}$. Polychaeta was the dominant taxonomic group $(81 \%, 17)$, followed by crustaceans $(19 \%, 4)$. No other groups or 'uniques' were recorded in this Site (Table 2). The lowest species count was recorded at Site 4. Abundances increased from east to west. Low $d$ and $H^{\prime}$ were recorded at Stn 10, and greatest $d$ and $H^{\prime}$ occurred at Stn 11 (Table 5). Mean species diversity was 2.48. ANOSIM results (Table 6) showed significant differences from all other sites (Site 1: $R=0.828, \mathrm{p}=$ $1.3 \%$; Site 2: $R=0.645, \mathrm{p}=2.9 \%$; Site 3: $R=0.691, \mathrm{p}=$ $4.8 \%$ and Site $5: R=0.563, \mathrm{p}=1.5 \%$ ). SIMPER analysis showed the Amphipods (Crustacea) Wildus parathambaroo and Ampelisca melanesiensis contributing the highest proportions to within group similarity $(21.9 \%$ each), followed by the polychaete Notomastus sp. 2 (15.6\%, Table 3).

\section{Site 5: Nadi Bay}

A total of 170 species of benthic fauna and 4684 individuals were recorded at Site 5 (Table 2), with 42 $(52 \%)$ of the species being 'uniques'. The mean density was 1040.8 ind. $\mathrm{m}^{-2}$. Polychaetes were the dominant group $(39 \%, 66)$ followed by crustaceans $(37 \%$, 63). Molluscs and echinoderms made up the remaining $21 \%$ (36) and $3 \%$ (5) of the population, respectively (Table 4). Greatest species counts were recorded at this site, with highest numbers occurring at stations furthest from the shore. Diversity measures (Table 5) indicated low species richness $(d)$ and diversity $\left(H^{\prime}\right)$ at Stn 39. Greatest $d$ and $H^{\prime}$ were recorded at Stn 40. A mean species diversity of 3.26 was recorded. The ANOSIM (Table 6) indicated no significant difference 



Fig. 7. Sample-species accumulation curves of the 5 sites (see Fig. 1) showing estimated species richness values (solid lines) with 95\% confidence intervals (dashed lines). Different numbers of stations have been sampled at each site, and species accumulations graphs with a more concave-downward curve demonstrate better sampling

from Sites $1(R=0.434, \mathrm{p}=0.1 \%), 2(R=0.321, \mathrm{p}=$ $0.1 \%)$ and $3(R=0.181, \mathrm{p}=9.6 \%)$, but did show significant difference from Site $4(R=0.563, \mathrm{p}=1.5 \%)$. SIMPER analysis (Table 3 ) revealed that largest contribution to within group similarity was made by the crustacean Cymadusa sp. (13.3\%), followed by the polychaetes Horstileanira sp. (8.5\%) and Magelona sp. $(7.6 \%)$.

\section{Environmental factors}

\section{Site 1: Suva Harbour}

Across all stations sampled at this site, the predominant grain size was $63 \mu \mathrm{m}$ (Fig. 9, Table 2). Stations situated within the reef passage at Site 1 had an abundance of poorly sorted, silty particles. The greatest amount of silt recorded at this site was $83.5 \%$, while the lowest was $46.9 \%$. Water depth increased from the north (close to the shore) to the reef. The deepest station was $113 \mathrm{~m}$ and the shallowest was $14 \mathrm{~m}$. Carbonate content increased with depth. The highest percentage of carbonate content was $81.8 \%$, while the lowest value was $25.0 \%$. Greatest organic content was recorded at stations closest to the shore and just inside the channel. Values ranged from 5.7 to $15.2 \%$. BIOENV analysis revealed that depth, distance from the reef and shoreline, and carbonate content had the greatest correlation (0.552) with the faunal composition.

Site 2: Laucala Bay Lagoon

The commonest grain size fraction was $63 \mu \mathrm{m}$ (Fig. 9, Table 2). The middle of Laucala Bay lagoon (Site 2) contained poorly sorted sediment, while closer to shore there was a change to moderately sorted sediment. Clay sediment was predominant at $53.0 \%$ of the stations located on either side of the channel, and silty sediment was found at the remaining stations in the middle of the lagoon and within the channel. The highest percentage of silt recorded at this site was $68.7 \%$, and the highest percentage of clay was $57.5 \%$. The stations closer to shore were shallower than those located close to the barrier reef. The deepest station at this site was $41 \mathrm{~m}$ and the shallowest $13 \mathrm{~m}$. Grain size and carbonate content were directly related to depth measurements. The carbonate values fell between 87.2 and $21.3 \%$. Stations sampled in the centre of the lagoon contained large percentages of organic matter, ranging between $5.8 \%$ and $27.7 \%$. Water depth and mean grain size had the highest correlation (0.348) with faunal composition at Site 2 (BIOENV analysis). 


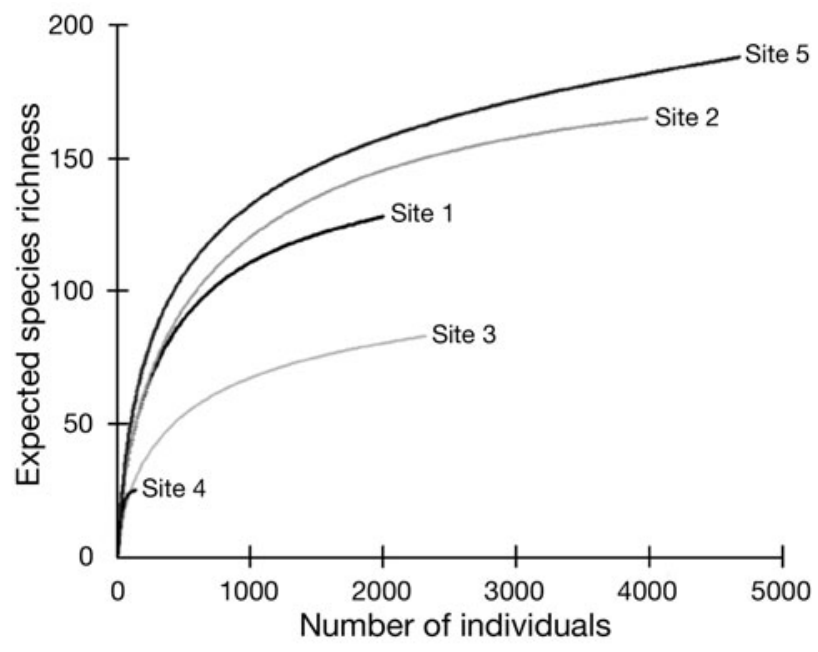

Fig. 8. Rarefaction curves for benthic fauna in the 5 sites (see Fig. 1) around Viti Levu indicating sites where sufficient individuals have been sampled in a community to determine species diversity

\section{Site 3: Rewa River Estuary}

The dominant grain size fraction at Site 3 (Rewa River Estuary) was $63 \mu \mathrm{m}$ (Fig. 9, Table 2), and there were moderate amounts of sorted silty sediment. The highest percentage of silt was 77.8\%. Depth increased at stations further away from the shore, with the greatest at $40 \mathrm{~m}$ and the shallowest at $24 \mathrm{~m}$. Terrigenous content and organic matter were high at the majority of stations. Terrigenous content ranged from 24.5 to $87.4 \%$ while organic content varied from 6.77 to $10.5 \%$. The sediment parameter skewness, with a correlation value of 0.663 best explained the faunal patterns at this site (BIO-ENV analysis).

\section{Site 4: Nukubuco Reef drop-off}

The Fore-Reef site situated close to the barrier reef platform contained large percentages of very coarse particles (>1 mm) that were well sorted (Fig. 9, Table 2). Site 4 sediment largely comprised clay; the highest amount was $82.4 \%$. The deepest stations were sampled at this site (213 and $123 \mathrm{~m}$ ), and depth increased westwards. Carbonate content followed a similar trend, and values of 96.7 and $93.3 \%$ were recorded. The organic content was greatest at the deeper station (7.1\%). BIOENV analysis was not possible at this site due to the small number of stations sampled.

\section{Site 5: Nadi Bay}

Stations at Site 5 contained predominantly finegrained $(63 \mu \mathrm{m})$ particles (Fig. 9, Table 2). Poorly sorted and silty sediment was most common, reaching a maximum value of $81.0 \%$. Stations were in general shallower than those on the eastern side of the island. The deepest station was at $39 \mathrm{~m}$, while the shallowest station was at $10 \mathrm{~m}$. Stations sampled within the bay were shallower, and depth increased away from the shore-line. Carbonate content was directly related to depth measurements, while high organic content was recorded at stations closer to the shore. The values for carbonate content were between 98.4 and $27.2 \%$, while organic content ranged from 4.6 to $11.0 \%$. The subset of environmental variables which best 'explained' the faunal patterns included distance from river, organic content and mean grain size (correlation value: 0.560, BIO-ENV)

\section{DISCUSSION}

\section{Community structure}

The Viti Levu (Fiji) soft sediment fauna was characterised by high biodiversity and large numbers of individuals, the exception being Site 3 (Rewa River Estuary), suggesting an unstable environment. Direct comparison of soft bottom community biodiversity of Viti Levu with those reported from previous studies conducted in the South Pacific is difficult because of the differences in methodology and habitats investigated. The total of 230 species collected in this study was higher than those recorded at the Great Astrolabe Reef, Fiji by Newell \& Clavier (1997) (207 taxa) and Schlacher et al (1998) (211 taxa), and along the Great

Table 4. Biological characteristics of the 5 sites (see Fig. 1)

\begin{tabular}{|c|c|c|c|c|c|c|c|c|}
\hline \multirow[t]{2}{*}{ Site } & \multicolumn{3}{|c|}{ - Total no. } & \multirow{2}{*}{ Polychaeta } & \multirow{2}{*}{$\begin{array}{l}\text { No. of } \\
\text { Crustacea }\end{array}$} & \multirow{2}{*}{$\begin{array}{l}\text { Mpecies } \\
\text { Mollusca }\end{array}$} & \multirow[b]{2}{*}{ Echinodermata } & \multirow{2}{*}{$\begin{array}{c}\text { Total no } \\
\text { of spp. }\end{array}$} \\
\hline & Stations & Samples & Individuals & & & & & \\
\hline 1 & 11 & 33 & 2010 & 55 & 35 & 20 & 3 & 113 \\
\hline 2 & 17 & 51 & 3985 & 55 & 63 & 17 & 3 & 138 \\
\hline 3 & 5 & 15 & 2323 & 38 & 21 & 8 & 3 & 70 \\
\hline 4 & 2 & 6 & 136 & 17 & 4 & 0 & 0 & 21 \\
\hline 5 & 15 & 45 & 4684 & 67 & 63 & 36 & 5 & 171 \\
\hline
\end{tabular}


Barrier Reef, Australia (154 taxa) by Riddle (1988). Frouin \& Hutchings (2001), however, collected 315 taxa in Tahitian lagoons and Chardy et al. (1988) recorded 311 taxa in New Caledonian lagoons. We found faunal den-sities of 226 to 1548 ind. $\mathrm{m}^{-2}$. Densi-

Table 5. Diversity indices for all 50 stations in 5 sites (see Fig. 1). N: total number of individuals, S: total number of species (richness), $H^{\prime}$ : Shannon-Weiner diversity index, $J^{\prime}$ : Pielou's evenness index, $d$ : Margalef's richness index

\begin{tabular}{|c|c|c|c|c|c|c|}
\hline Stn & Site & $\mathrm{N}$ & $\mathrm{S}$ & $J^{\prime}$ & $H^{\prime}$ & $d$ \\
\hline 25 & 1 & 104 & 25 & 0.89 & 2.86 & 5.17 \\
\hline 26 & 1 & 272 & 70 & 0.89 & 3.78 & 12.31 \\
\hline 27 & 1 & 241 & 39 & 0.89 & 3.28 & 6.93 \\
\hline 28 & 1 & 171 & 41 & 0.87 & 3.21 & 7.58 \\
\hline 29 & 1 & 165 & 43 & 0.88 & 3.31 & 8.23 \\
\hline 30 & 1 & 116 & 33 & 0.88 & 3.09 & 6.73 \\
\hline 31 & 1 & 181 & 48 & 0.87 & 3.34 & 8.85 \\
\hline 32 & 1 & 136 & 40 & 0.86 & 3.15 & 7.73 \\
\hline 33 & 1 & 205 & 48 & 0.86 & 3.32 & 8.64 \\
\hline 34 & 1 & 225 & 38 & 0.77 & 2.76 & 6.46 \\
\hline 35 & 1 & 194 & 40 & 0.82 & 3.02 & 7.40 \\
\hline 1 & 2 & 34 & 15 & 0.86 & 2.39 & 4.25 \\
\hline 2 & 2 & 116 & 28 & 0.82 & 2.74 & 5.68 \\
\hline 3 & 2 & 142 & 48 & 0.93 & 3.59 & 9.48 \\
\hline 4 & 2 & 382 & 51 & 0.76 & 2.99 & 8.24 \\
\hline 5 & 2 & 277 & 59 & 0.83 & 3.38 & 10.14 \\
\hline 6 & 2 & 208 & 45 & 0.86 & 3.27 & 8.24 \\
\hline 7 & 2 & 189 & 54 & 0.81 & 3.19 & 9.54 \\
\hline 8 & 2 & 269 & 50 & 0.84 & 3.29 & 8.58 \\
\hline 9 & 2 & 120 & 30 & 0.84 & 2.82 & 5.85 \\
\hline 12 & 2 & 327 & 46 & 0.78 & 2.94 & 7.43 \\
\hline 13 & 2 & 251 & 33 & 0.67 & 2.31 & 5.61 \\
\hline 14 & 2 & 139 & 21 & 0.74 & 2.29 & 4.26 \\
\hline 15 & 2 & 216 & 38 & 0.83 & 3.02 & 6.88 \\
\hline 16 & 2 & 317 & 64 & 0.86 & 3.59 & 10.94 \\
\hline 17 & 2 & 450 & 81 & 0.87 & 3.82 & 12.77 \\
\hline 18 & 2 & 187 & 42 & 0.86 & 3.22 & 7.84 \\
\hline 19 & 2 & 361 & 50 & 0.83 & 3.21 & 7.98 \\
\hline 20 & 3 & 50 & 51 & 0.79 & 3.10 & 8.19 \\
\hline 21 & 3 & 33 & 35 & 0.78 & 2.73 & 5.65 \\
\hline 22 & 3 & 38 & 38 & 0.79 & 2.86 & 6.42 \\
\hline 23 & 3 & 23 & 22 & 0.17 & 0.53 & 3.17 \\
\hline 24 & 3 & 35 & 36 & 0.79 & 2.82 & 6.04 \\
\hline 10 & 4 & 8 & 7 & 0.90 & 1.87 & 1.87 \\
\hline 11 & 4 & 25 & 25 & 0.96 & 3.10 & 5.28 \\
\hline 36 & 5 & 64 & 64 & 0.77 & 3.21 & 9.43 \\
\hline 37 & 5 & 70 & 71 & 0.89 & 3.82 & 12.65 \\
\hline 38 & 5 & 27 & 27 & 0.89 & 2.93 & 5.14 \\
\hline 39 & 5 & 16 & 16 & 0.93 & 2.58 & 3.47 \\
\hline 40 & 5 & 108 & 107 & 0.83 & 3.87 & 14.70 \\
\hline 41 & 5 & 61 & 65 & 0.89 & 3.65 & 11.01 \\
\hline 42 & 5 & 28 & 29 & 0.87 & 2.90 & 6.09 \\
\hline 43 & 5 & 28 & 29 & 0.89 & 2.97 & 5.66 \\
\hline 44 & 5 & 17 & 17 & 0.95 & 2.69 & 3.83 \\
\hline 45 & 5 & 80 & 82 & 0.86 & 3.78 & 13.16 \\
\hline 46 & 5 & 32 & 32 & 0.89 & 3.09 & 6.42 \\
\hline 47 & 5 & 92 & 95 & 0.87 & 3.92 & 14.20 \\
\hline 48 & 5 & 29 & 29 & 0.82 & 2.76 & 5.92 \\
\hline 49 & 5 & 52 & 54 & 0.94 & 3.70 & 10.46 \\
\hline 50 & 5 & 26 & 25 & 0.92 & 3.01 & 5.63 \\
\hline
\end{tabular}

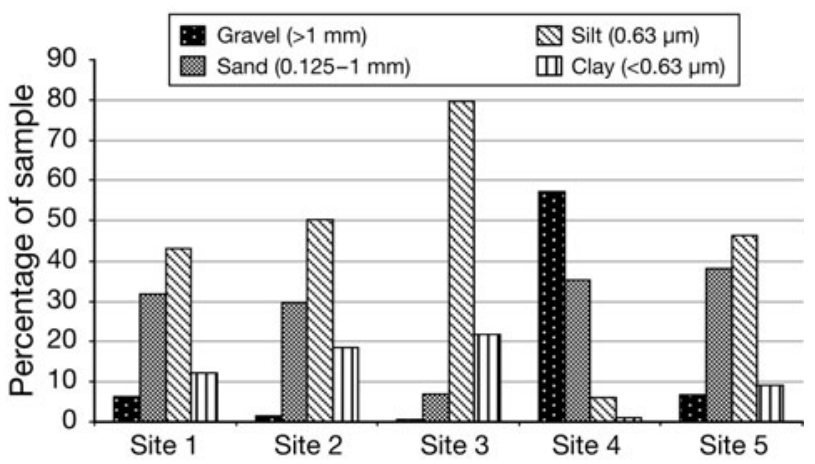

Fig. 9. Relative distributions of sediment particle sizes of gravel, sand, silt or clay at 5 sites (see Fig. 1); bars are averages of stations within sites

ties of 3115 to 43690 ind. $\mathrm{m}^{-2}$ (using a $0.5 \mathrm{~mm}$ sieve) were recorded on the central Great Barrier Reef (Riddle 1988), 41 to 220 ind. $\mathrm{m}^{-2}$ in New Caledonia (Chardy et al. 1988) and 22 to 868 ('large') and 670 to 20818 ('small') ind. $\mathrm{m}^{-2}$ in Tahiti (Frouin \& Hutchings 2001). Similar values of 870 to 10260 ind. $\mathrm{m}^{-2}$ were measured in Hawaii (DeFelice \& Parrish 2001) and in French Polynesia (496 to 4866 ind. $\mathrm{m}^{-2}$; Thomassin et al. 1982). These figures fall within the range given by Alongi (1989) for the southern Pacific (307 to 16750 ind. $\mathrm{m}^{-2}$ ). Neither the species accumulation curves nor the Jackknife 1 and 2 nor bootstrap estimates reached asymptotes, indicating insufficient sampling in our program. A solution would be to increase sampling intensity, but Schlacher et al (1998) and Ellingsen (2002) both agree that with increasing sample size, rare species are continuously added, but those with low abundance would have a low probability of being recorded. Hence, an asymptote would not be reached. On the contrary however, increased sampling and research in invertebrate fauna would highlight more rare and endemic species in Fiji, as shown in New Caledonia. The wealth of inventory and systematic work conducted on the New Caledonian fauna in recent years has revealed high levels of regional endemicity and it is likely a similar trend would be observed in Fiji were a similar research intensity applied.

Common species were widely spatially distributed in Vitl Levu, while species of low abundance had compressed range sizes (Table 1), as shown previously by Schlacher et al (1998) and Ellingsen (2002). This generally observed pattern can be explained by inadequate sampling, which underestimates range sizes. Polychaetes were the most common taxonomic group and had the highest proportion of widely distributed species followed by crustaceans and molluscs. Echinoderms were more restricted in their distributions across the 5 sites. The 'uniques' comprised a significant fraction of the benthos recorded (35\%). Similar proportions were found by Schlacher et al. (1998) in the Great 
Table 6. One-way analysis of similarity (ANOSIM) for all 5 sites. Sample statistic (Global $R$ ): 0.343

\begin{tabular}{|c|c|c|c|c|c|c|}
\hline Site location (Site no.) & $\begin{array}{c}R \\
\text { statistic }\end{array}$ & $\begin{array}{c}\text { Significance } \\
\text { level \% }\end{array}$ & $\begin{array}{l}\text { Significant } \\
\text { difference }\end{array}$ & $\begin{array}{c}\text { Possible } \\
\text { permutations }\end{array}$ & $\begin{array}{c}\text { Actual } \\
\text { permutations }\end{array}$ & $\begin{array}{l}\text { No. } \\
\text { obs. }\end{array}$ \\
\hline Suva Harbour (1), Laucala Bay (2) & 0.242 & 0.3 & No & 21474180 & 999 & 2 \\
\hline Suva Harbour (1), Rewa River (3) & 0.565 & 0.2 & No & 4368 & 999 & 1 \\
\hline Suva Harbour (1), Fore-Reef (4) & 0.828 & 1.3 & No & 78 & 78 & 1 \\
\hline Suva Harbour (1), Nadi Bay (5) & 0.434 & 0.1 & Yes & 7726160 & 999 & 0 \\
\hline Laucala Bay (2), Rewa River (3) & 0.159 & 17.1 & No & 26334 & 999 & 170 \\
\hline Laucala Bay (2), Fore-Reef (4) & 0.645 & 2.9 & No & 171 & 171 & 5 \\
\hline Laucala Bay (2), Nadi Bay (5) & 0.321 & 0.1 & Yes & 565722720 & 999 & 0 \\
\hline Rewa River (3), Fore-Reef (4) & 0.691 & 4.8 & No & 21 & 21 & 1 \\
\hline Rewa River (3), Nadi Bay (5) & 0.181 & 9.6 & No & 15504 & 999 & 95 \\
\hline Fore-Reef (4), Nadi Bay (5) & 0.563 & 1.5 & No & 136 & 136 & 2 \\
\hline
\end{tabular}

Astrolabe Reef lagoon (Fiji, 42\%), by Ellingsen (2001, 2002) off Norway (39\%) and Bouchet et al (2002) off new Caledonia (32\%), suggesting that high spatial heterogeneity in community structure is a feature of coral reef environments.

Diversity and abundance increased from west to east across the study sites. Species diversity and richness were greatest at Site 5 (west of Viti Levu), and along the coast to the southeast of the island, species diversity and richness decreased. The number of 'uniques' correlated with species richness. Site 5 had the greatest species richness (49.5) and proportion of 'uniques' (52\%). The fore-reef area (Site 4 ) had lowest species richness (16) and no 'uniques'. Likewise low species richness was found in Site 3 (36.4), and $7.4 \%$ of the fauna were 'uniques'. These 2 sites are subjected to regular high wave action and large salinity fluctuations. Gray (2002) reported that low species richness occurs in habitats subjected to constantly fluctuating environmental conditions. Ellingsen (2001) also reported that sites with a low proportion of restrictedrange species also had low species richness.

Community structure at the 5 sites shows some similarities. In the MDS ordination (Fig. 3), 3 distinct clusters can be seen. The fauna at Site 5 is characterised by high diversity and resembles biologically accommodated communities found in stable environments (Shin \& Thompson 1982). Sites 1 and 2 both had very high number of individuals and high diversity. A previous study at these 2 locations similarly found Site 1 more diverse than Site 2 (Corless 1995). Sites 3 and 4 had very low numbers of individuals and low diversities. Khan et al (2004) reported that in a healthy environment, the Shannon diversity index is usually high (2.5 to 3.5). The values for Sites 3 and 4 are lower, suggesting unstable environments. The distinct clusters on the MDS ordination (Fig. 3) are most likely due to the presence and absence of species from the sites. SIMPER analysis (Tables 2) shows species that caused most similarity within sites.

\section{Factors influencing community structure}

Granulometric analyses showed slight variations among sites, but the general trends are apparent (Fig. 9). Sediment compositions among Sites 1, 2 and 5 were very similar. Great importance has been placed on soft bottom sediments and sediment grain size as key factor in determining community differences (McIntyre 1969, Driscoll 1975, Hily et al. 1992, Snelgrove \& Butman 1994, Frouin 2000). The difference in species richness and diversity between Sites 1, 2 and 5 suggests that sediment grain size did not affect infaunal community structure. The presence of heterogenous faunal communities among stations and sites of similar sediment type is not congruent with a concept of distinct associations between benthic community and sediment types. However the markedly lower species diversity in Site 3 fits the general observation that silt may be a more difficult sediment to colonise and coarser sediments are able to support more life (Gray 1974, Shin \& Thompson, 1982).

The fluctuation in available organic matter is thought to be one of the principal causes of faunal change in near-shore benthic environments (Pearson \& Rosenberg 1978, Gee \& Warwick 1985, Linton \& Taghon 2000). However, we found no clear correlation between benthic community structure and organic content. Greater organic contents were recorded at Sites 1,2 and 3, which may be due to the influence of the Rewa River and the mangroves found along these study sites. Hansen et al. (1992) have reported that detrital particles from seagrass and mangroves may serve as a source of organic matter in lagoons. The degree of lagoon closure, nutrient input rates, mixing rates within the lagoon and the residence time of lagoonal waters at Sites 1, 2 and 3 may be related to the high levels of organic content recorded here. The lack of a barrier reef at Site 5, and the location of Site 4 outside the barrier reef likely 
increases nutrient flushing rate, resulting in low organic content.

BIO-ENV showed that depth, distance from reef and river, and sand content (0.397) were most closely related to community structure. Studies have shown (Hutchings \& Wells 1993, Glover et al. 2002) that factors like current energy, productivity and terrigenous input become important with increasing depth in determining benthic communities. The clusters formed in the PCA plot (Fig. 4) show 3 groupings. The close proximity of Site 3 to 1 and 2 in the cluster is most probably due to the influence of the Rewa River and tidal regimes experienced by these 3 sites on the southeastern side of the island. This could also explain the distinct separation of Site 5 (which is situated on the western side of the island) from those situated on the southeastern coast. The results show weak relationships between some of the environmental variables and faunal composition. Schlacher et al. (1998) and Frouin \& Hutchings (2001) also found no clear relationship between faunal assemblage and environmental variables in a similar coral lagoon, suggesting factors other than those recorded might be influencing community structure. Ellingsen (2002) suggests that in any given locality, a number of interacting factors will be involved in determining its community structure. Vroom et al. (2005) found geological and oceanographic features (distance from reef, islands) lead to spatially variable environmental regimes (water flow, turbidity, temperature). This variability could explain the biological heterogeneity found among the sites.

Local oceanic conditions have been suggested to play a role in observed diversity gradients (Moyer et al. 2003). Site 1 (Suva Harbour) is a shallow embayment with an estuarine-type circulation (Corless 1995). High diversity recorded here could be a result of maximum tidal flushing experienced in this area. Site 2 (Laucala Bay Lagoon) is a large area which lies between the Suva peninsula and Rewa Delta. The bay is protected by coral reefs, which are exposed at low tides, allowing seawater to enter over the reef twice daily during high tide (Naidu et al.1991). Two narrow reef passages on either side of the bay also provide limited circulation. Site 5, which was the most diverse, has a fringing reef that does not enclose the bay and has sporadic patch reefs. These formations allow unobstructed circulation of oceanic water. Although not investigated in this study, the tidal current regime of lagoons around Fiji could help determine if changes in the current in an area would lead to changes in community structure.

Run offs, sewer outfalls, and rivers are likely to increase nutrient availability and sedimentation, which can lead to decreased diversity (Frouin 2000, Moyer et al. 2003). The presence of a sewage outfall in Laucala Bay Lagoon is likely to increase nutrient avail- ability (Naidu et al. 1991, Corless 1995). We found higher levels of organic matter and lower benthic diversity closest to the outfall. Water quality, turbidity, salinity and temperature may play roles in determining community structure. Unfortunately, due to time and economic constraints these parameters could not be recorded for all sites. However, results that were collected show highest salinity and temperature at Site 1 and highest turbidity at Site 3.

\section{CONCLUSION}

Benthic communities in the sites around the Fiji lagoons are rich and diverse; species numbers and abundances are within the ranges observed in Australia, New Caledonia and Tahiti. Benthic communities showed spatial variability in abundance and taxonomic richness. The number of 'uniques' were comparable to those found in similar studies, suggesting a degree of 'spot endemism'. The suggestion of Roberts et al. (2002) that biodiversity decreases eastward across the Pacific was not supported, possibly because of inadequate sampling intensity and extent in the past. Fiji has been identified by the scientific community as having significant components of 'Outstanding Universal Value' in its tropical coastal, marine and small island biodiversity; further studies have been recommended to ascertain which components are of World Heritage value (The Hanoi Statement, 2002, available at: http://whc.unesco.org/uploads/events/ documents/event-501-1.pdf)

Stations with similar environmental variables did not always have the same community structure. As in other studies (Schlacher et al 1998, Ellingsen 2002, Fonseca \& Netto 2006), no single, well-defined relationship was observed between environmental factors and biotic assemblages. It would appear that in any given location a number of different interacting biological and physical factors are involved in determining the distribution and abundance of benthos (Snelgrove \& Butman 1994, Frouin 2000, Ellingsen 2002. Further research on other environmental variables (chlorophyll $a$, salinity, temperature, turbidity) is needed in attempting to discern strong relationships between community structure and environment.

With increasing population, urban development and mounting anthropogenic pressures in coastal areas of Fiji, there is an increasing need to understand the mechanisms influencing biodiversity, and to develop conservation strategies. Site 3 had low diversity and dominance of a single species, indicative of a perturbed (organically enriched) environment. Pearson \& Rosenberg (1978) and Lee et al. (2006) found that initial organic enrichment results in higher diversity val- 
ues, followed subsequently by decreases as organic enrichment continually increases. The lagoons are a vital economic asset to the Fiji islanders and maintaining the biodiversity of the benthic fauna is a crucial part of maintaining this asset. In order to accurately gauge the biodiversity of benthic fauna in Fiji and to maintain native levels of biological diversity, more regular surveys and studies are needed and the results incorporated into a monitoring program to assess the effects of the changing environment.

Acknowledgements. This study was made possible by funding from the French Embassy (Suva, Fiji). We thank P. Newell, B. Richer de Forges, colleagues at IRD (Noumea, New Caledonia) and from the RV 'Alis'. Many thanks also go to T. Schlacher, J. Seeto, and P. Hutchings for their assistance in identification of fauna and data analysis.

\section{LITERATURE CITED}

Alongi DM (1989) The role of soft sediment benthic communities in tropical mangroves and coral reef ecosystems. CRC Crit Rev Aquat Sci 1:243-280

Alongi DM (1990) The ecology of tropical soft-bottom benthic ecosystems. Oceanogr Mar Biol Annu Rev 28:381-496

Bouchet P, Lozouet P, Maestrati P, Heros V (2002) Assessing the magnitude of species richness in tropical marine environments: exceptionally high numbers of molluscs at a New Caledonia site. Biol J Linn Soc 75:421-436

Chardy P, Chevillon C, Clavier J (1988) Major benthic communities of the south-west lagoon of New Caledonia. Coral Reefs 7:69-75

> Clarke KR, Ainsworth M (1993) A method of linking multivariate community structure to environmental variables. Mar Ecol Prog Ser 92:205-219

Clarke KR, Green RH (1988) Statistical design and analysis for a 'biological effects' study. Mar Ecol Prog Ser 46: 213-226

Clarke KM, Warwick RM (1994) Changes in marine communities: an approach to statistical analysis and interpretation. Natural Environmental Research Council, Plymouth

Clarke KR, Warwick RM (2001) Change in marine communities: an approach to statistical analysis and interpretation. Primer-E, Plymouth Marine Laboratory, Plymouth

Colwell RK (1999) Estimates: statistical estimation of species richness and shared species from samples, Ver 5. Available at: http://viceroy.eeb.uconn.edu/estimates

Coppard SE, Campbell AC (2005) Distribution and abundance of regular sea urchins on two coral reefs in Fiji. Micronesica 37(2):249-269

Corless M (1995) An exploratory study of marine pollution in Suva Harbour and Laucala Bay (Fiji) using benthic community structure analysis. IAS Environmental Report No. 79. University of the South Pacific, Suva

Day PR (1965) Particle fractionation and particle-size analysis. In: Black CA (ed) Methods of soil analysis, Part 1. American Society of Agronomy, Madison, WI, p 545-567

> DeFelice RC, Parrish JD (2001) Physical processes dominate in shaping invertebrate assemblages in reef-associated sediments of an exposed Hawaiian coast. Mar Ecol Prog Ser 215:121-131
Driscoll EG (1975) Sediment-animal-water interaction, Buzzards Bay, Massachusetts. J Mar Res 33:275-302

Ellingsen KE (2001) Biodiversity of a continental shelf softsediment macrobenthos community. Mar Ecol Prog Ser 218: 1-15

Ellingsen KE (2002) Soft sediment benthic biodiversity on the continental shelf in relation to environmental variability. Mar Ecol Prog Ser 232:15-27

Folk RL (1974) Petrology of sedimentary rocks. Hemphill Publishing, Austin, TX

- Fonseca G, Netto SA (2006) Shallow sublittoral benthic communities of the Laguna Estuarine System, South Brazil. Braz J Oceanogr 54:41-45

- Frouin P (2000) Effects of anthropogenic disturbances of tropical soft-bottom benthic communities. Mar Ecol Prog Ser 194:39-53

Frouin P, Hutchings P (2001) Macrobenthic communities in a tropical lagoon (Tahiti, French Polynesia, Central Pacific). Coral Reefs 19(3):277-285

> Gee MJ, Warwick M (1985) Effects of organic enrichments on meiofaunal abundance and community structure in sublittoral soft sediments. J Exp Mar Biol Ecol 91(3):247-262

> Glover AG, Smith CR, Paterson GLJ, Wilson GDF, Hawkins L, Sheader M (2002) Polychaete species diversity in the central Pacific abyss: local and regional patterns, and relationships with productivity. Mar Ecol Prog Ser 240: $157-170$

Gray JS (1974) Animal-sediment relationships. Oceanogr Mar Biol Annu Rev 17:223-261

Gray JS (1997) Marine biodiversity: patterns, threats and conservation needs. Biodivers Conserv 6:153-175

Gray JS (2002) Species richness of marine soft sediments. Mar Ecol Prog Ser 244:285-297

> Hansen JA, Klumpp DW, Alongi DM, Dayton PK, Riddle MJ (1992) Detrital pathways in a coral reef lagoon. II. Detritus deposition, benthic microbial biomass and production. Mar Biol 113:363-372

> Hily C, Potin P, Floc'h JY (1992) Structure of subtidal algal assemblages on soft-bottom sediments: fauna/flora interactions and role of disturbances in the Bay of Brest, France. Mar Ecol Prog Ser 85:115-130

Hutchings PA, Wells FE (1993) An analysis of the marine invertebrate community at Hoi Ha Wan, Hong Kong. In: Morton B (ed) The marine flora and fauna of Hong Kong and southern China III. Proc 4th Int Mar Biol Workshop: The marine flora and fauna of Hong Kong and southern China. Hong Kong University Press, Hong Kong, p 851-864

- Keough MJ, Quinn GP (1991) Causality and the choice of measurements for detecting human impacts in marine environments. Aust J Mar Freshw Res 42:539-554

Khan SA, Murugesan P, Lyla PS, Jaganathan S (2004) A new indicator macro invertebrate of pollution and utility of graphical tools and diversity indices in pollution monitoring studies. Curr Sci 87:1508-1510

> Lee HW, Bailey-Brock JH, McGurr MM (2006) Temporal changes in the polychaete infaunal community surrounding a Hawaiian mariculture operation. Mar Ecol Prog Ser 307:175-185

Linton D, Taghon GL (2000) Feeding, growth and fecundity of Capitella sp.1 in relation to sediment organic concentration. Mar Ecol Prog Ser 205:229-240

> McIntyre AD (1969) Ecology of marine meiobenthos. Biol Rev Camb Philos Soc 44:245-290

> Moyer RP, Riegl B, Banks K, Dodge RE (2003) Spatial patterns and ecology of benthic communities on a high-latitude South Florida (Broward County, USA) reef system. Coral Reefs 22:447-464 
Naidu S, Aalbersberg WG, Brodie JE, Fuavao VA and others (1991) Water quality studies on selected South Pacific lagoons. UNEP Regional Seas Reports and Studies, No. 136. South Pacific Regional Environmental Programs. UNEP, Geneva

Nair V (2003) Fiji Island Marine Ecoregion. An overview of understanding biodiversity, threats, opportunities and key stakeholders for conservation. WWF Fiji Programme, Suva. Available at: conserveonline.org/workspaces/ era. peer.review/workshops/9thbrisbane/workplans/FIME\%20 Reconnaissance.pdf

Newell P, Clavier J (1997) Quantitative structure of soft substrate macrobenthos of Fiji's Great Astrolabe Lagoon. Proc 8th Int Coral Reef Symp 1:455-458

Pearson TH, Rosenberg R (1978) Macrobenthic succession in relation to organic enrichment and pollution of the marine environment. Oceanogr Mar Biol Annu Rev 16: 229-311

Reaka-Kudla ML (1996) The global biodiversity of coral reefs: a comparison with rain forests. In: Reaka-Kudla ML, Wilson DE, Wilson EO (eds) Biodiversity II. Joseph Henry Press, Washington, DC, p 83-108

Richer de Forges B (1991) Le benthos des fonds meubles des lagons de Nouvelle-Calédonie, Etudes et thèses Vol 1. ORSTOM, Paris

Riddle MJ (1988) Patterns in the distribution of macrofaunal communities in coral reef sediments on the central Great Barrier Reef. Mar Ecol Prog Ser 47:281-292

Roberts CM, McClean CJ, Veron JEN, Hawkins JP and others (2002) Marine biodiversity hotspots and conservation priorities for tropical reefs. Science 295:1280-1284

Schlacher TA, Newell P, Clavier J, Schlacher-Hoenlinger MA, Chevillion C, Britton J (1998) Soft-sediment benthic community structure in a coral reef lagoon - the prominence of spatial heterogeneity and 'spot endemism'. Mar Ecol Prog Ser 174:159-174

Schneider W, Schmelzer I, Wuertz J (1995) Sedimentological interplay of siliciclastic Rewa River input and organic carbonate production of the Suva Barrier Reef, Laucala Bay, Fiji. IMR, University of the South Pacific, Suva

Shin PKS, Thompson GB (1982) Spatial distribution of infaunal benthos of Hong Kong. Mar Ecol Prog Ser 10:37-47
Shorten GG (1993) The geological and tectonic settings for ground failure hazards in Suva Harbour environs. Memoir 3. Mineral Resources Department, Suva, p 49-55

> Smith EP, van Belle G (1984) Nonparametric estimation of species richness. Biometrics 40:119-129

Snelgrove PVR, Butman CA (1994) Animal-sediment relationships revisited: cause versus effects. Oceanogr Mar Biol Annu Rev 32:111-117

Terry J (1999) Investigating the use of the turbidity tube device for measuring suspended sediment concentration in humid tropical rivers - examples from Viti Levu Island, Fiji. S Pac J Nat Sci 18:36-40

Thomassin BA, Jouin C, Renaud-Mornant J, Richard G, Salvat B (1982) Macrofauna and meiofauna in the coral sediments on the Tiahura Reef complex, Moorea Island (French Polynesia). Tethys 10:392-397

Vroom PS, Kimberly NP, Kimberly AP, Kukea-Shultz JK (2005) Spatial heterogeneity of benthic community assemblages with an emphasis on reef algae at French Frigate Shoals, Northwestern Hawai'ian Islands. Coral Reefs 24: $574-581$

Vuki VC, Zann LP, Naqasima M, Vuki M (2000) The Fiji islands. In: Sheppard CRC (ed) Seas at the millenium: an environmental evaluation. Pergamon Press, Oxford, p 751-764

Warwick RM, Ruswahyuni (1987) Comparative study of the structure of some tropical and temperate marine soft-bottom macrobenthic communities. Mar Biol 95:641-649

Whippy-Morris C, Pratt C (1998) Marine Biodiversity Technical Group report. Fiji Biodiversity Strategy and Action Plan Project. Environment Department, Fiji Government, Suva

Spalding MD, Ravilous C, Green EP (2001) World atlas of coral reefs. University of California Press, Berkeley, CA

WRI (World Resources Institute) (1999). Status of coral reefs classified by potential threat from human activities. Status of the Worlds Coral Reefs: Pacific Ocean Page. Available at: http://archive.wri.org/pubs/pubs_content_print.cfm? ContentID=1338 (accessed January 2007)

WWF (2008) The coral triangle. Available at: http://assets. wwf.org.my/downloads/coral_triangle (accessed January 7 , 2007)

Submitted: September 3, 2007; Accepted: June 19, 2008

Proofs received from author(s): October 27, 2008
Editorial responsibility: Matthias Seaman, Oldendorf/Luhe, Germany 